The final published version of this article can be found at http://dx.doi.org/10.1016/j.tet.2007.03.027. 


\section{Graphical Abstract}

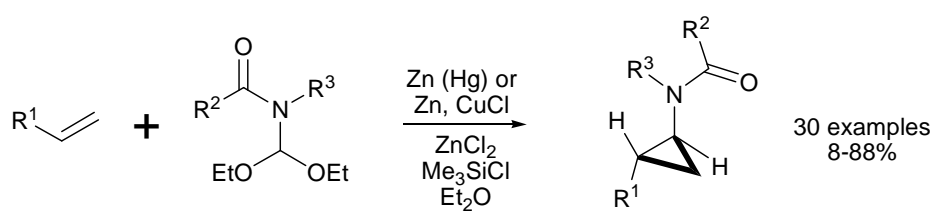




\title{
Observations on the direct amidocyclopropanation of alkenes using organozinc carbenoids
}

\author{
William B. Motherwell, ${ }^{*}{ }^{a}$ Guillaume Bégis, ${ }^{a}$ David E. Cladingboel, ${ }^{b}$ Laure Jerome ${ }^{a}$ \\ and Tom D. Sheppard.
}

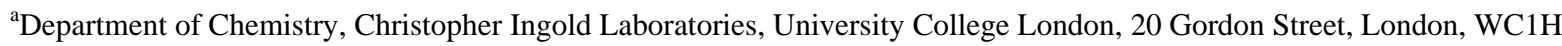
OAJ, UK.

${ }^{\mathrm{b}}$ AstraZeneca R\&D Charnwood, Bakewell Road, Loughborough, LE11 5RH, UK.

The aminocyclopropyl unit is present in a wide range of biologically active natural products and pharmaceuticals. Traditional approaches to the synthesis of aminocyclopropanes include the Curtius rearrangement of cyclopropylcarboxylic acids, ${ }^{2}$ the reduction of nitrocyclopropanes, ${ }^{3}$ the reductive amination of cyclopropanone acetals ${ }^{4}$ and the cyclopropanation of enamines. ${ }^{5}$ More recently, de Meijere has demonstrated a new titanium mediated cyclopropanation of amides or nitriles using 1,2-dimetallic derivatives of monosubstituted alkenes. ${ }^{6}$ By way of contrast however, the search for simple and experimentally convenient methods for the direct addition of heteroatom substituted carbenoids to unactivated alkenes has proven to be more elusive, with direct alkoxycyclopropanation and aminocyclopropanation requiring the use of such reagents as stoichiometric Fischer carbene complexes. As an extension of our earlier studies on the cyclopropanation of alkenes using organozinc carbenoids prepared from simple carbonyl compounds ${ }^{8}$ or their derived acetals, ${ }^{9}$ we have previously demonstrated that orthoformates are useful precursors for direct alkoxycyclopropanation. ${ }^{10}$ More recently, we have also shown that diethoxymethylpyrrolidinone can be used for generation of an amido substituted organozinc carbenoid, ${ }^{11}$ and that selection of a chiral oxazolidinone congener leads to an asymmetric version of this amidocyclopropanation reaction. ${ }^{22}$ In the present paper, we wish to report, in full, our detailed observations on the scope and limitations of simple achiral diethoxymethylamide carbenoid precursors for the construction of densely functionalised amidocyclopropanes.

From a mechanistic standpoint, we envisaged that the evolution of a suitably functionalised amidoorganozinc carbenoid from a diethoxymethylamide $\mathbf{1}$ could proceed as shown in Scheme 1. Thus, coordination of a Lewis acid (either $\mathrm{ZnCl}_{2}$ or $\mathrm{Me}_{3} \mathrm{SiCl}$ ) to one of the ethoxy groups of the amide $\mathbf{1}$ leads to the formation of cation $\mathbf{2}$ which can be reduced by two electron delivery from metallic zinc to give the organozinc derivative 3 . Lewis acid mediated $S_{N} 1$ displacement of the remaining ethoxy group by chloride then formally leads to the organozinc carbenoid which could exist as either the tetrahedral form $\mathbf{4}$ or the planar iminium form $\mathbf{5}$.
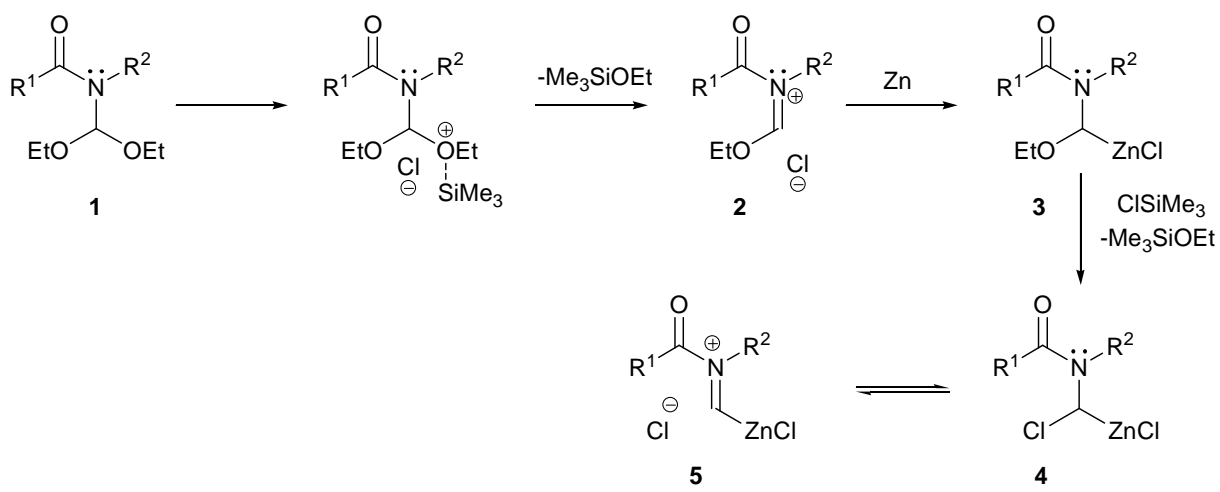

\section{Scheme 1}

The diethoxymethylamide carbenoid precursors 12-17 were initially prepared by heating the corresponding amides 6-11 in a large excess of triethyl orthoformate in the presence of a catalytic amount of aluminium chloride according to a literature procedure (Scheme 2 and Table 1). ${ }^{13}$ However, this method is inconvenient, particularly on larger scales, due to the difficulty in removing the large excess of orthoformate, and hence alternative methods were investigated. We found that the diethoxymethyl derivative of pyrrolidinone 6 (Entry 1) could 
be conveniently prepared on a $10 \mathrm{~g}$ scale using only 2.6 equivalents of triethyl orthoformate in the presence of chlorotrimethylsilane and a catalytic amount of triphenylphosphine at room temperature. After purification by distillation, the dialkoxymethyllactam $\mathbf{1 2}$ was obtained in 61\% yield. In a similar fashion, diethoxymethyloxazolidinone 13 (Entry 2) was obtained in $60 \%$ yield (10 g scale) using 2.6 equivalents of orthoester and a catalytic quantity of acetyl chloride. This latter procedure is the most convenient but was not generally applicable to the preparation of the other diethoxymethyl derivatives. It is notable that in this case the starting oxazolidinone is insoluble in the reaction mixture and gradually dissolves as the reaction progresses, and this gradual dissolution may well be important for the success of the reaction. The yields are essentially comparable to the $\mathrm{AlCl}_{3}$ catalysed reactions and these methods avoid the requirement for a large excess of triethyl orthoformate and the associated purification problems which then arise.

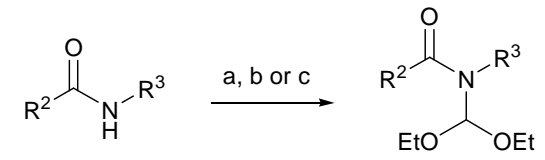

Scheme 2

a. $(\mathrm{EtO})_{3} \mathrm{CH}, \mathrm{AlCl}_{3}, 155^{\circ} \mathrm{C}$ (Method A). b. (EtO) ${ }_{3} \mathrm{CH}$, cat. $\mathrm{Me}_{3} \mathrm{SiCl}$, cat. $\mathrm{PPh}_{3}, \mathrm{CH}_{2} \mathrm{Cl}_{2}$, rt (Method B). c. $(\mathrm{EtO})_{3} \mathrm{CH}$, cat. $\mathrm{AcCl}$, rt (Method C).

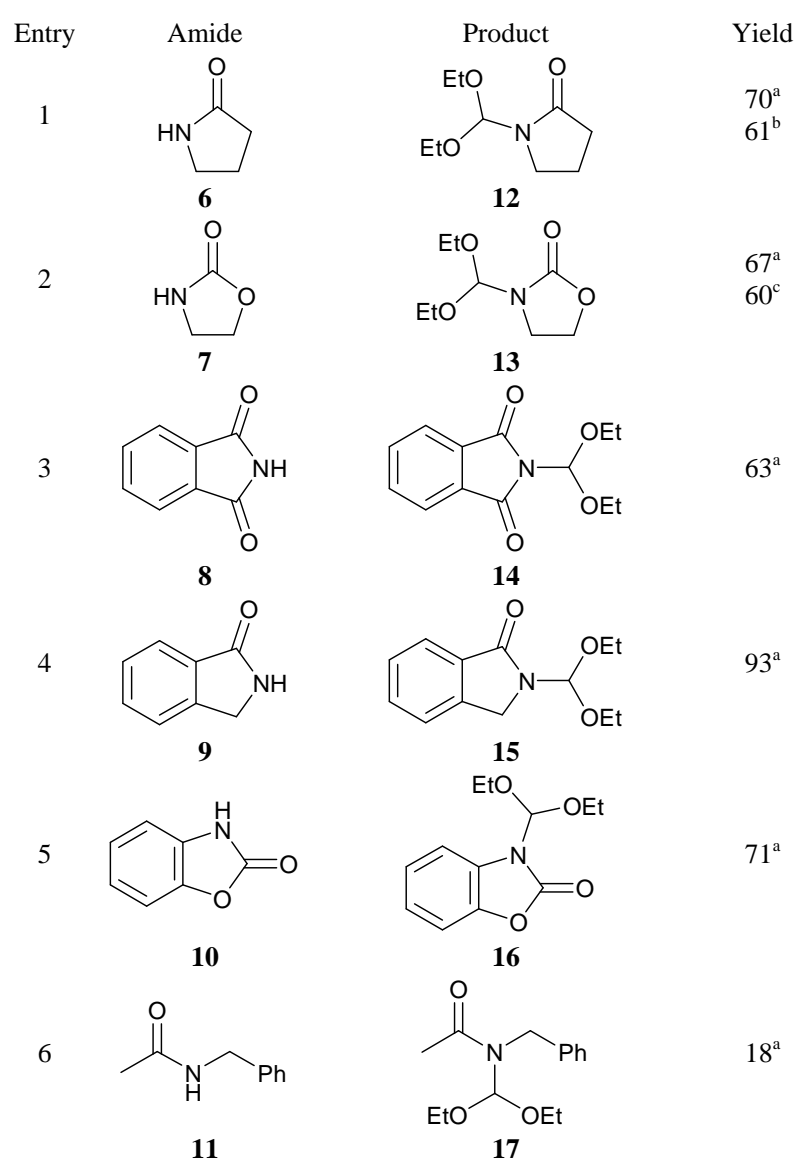

Table 1 a. Method A, b. Method B, c. Method C.

With the required diethoxymethylamides in hand, the amidocyclopropanation of a wide range of alkenes was investigated using zinc, chlorotrimethylsilane and zinc chloride (Scheme 3 and Table 2). In the initial reactions, zinc amalgam was employed as the metal source. Although easily handled, the amalgam is undesirable due to the toxicity and environmental issues associated with the use of mercury and its salts. Moreover, we have also observed that the zinc amalgam has a tendency to form hard spheres during the course of the cyclopropanation reactions which can lead to variable yields, presumably due to the large decrease in the available surface area of the metal. To this end, we were keen to investigate alternative metal 
sources, and the use of zinc dust, together with a catalytic quantity of copper (I) chloride, was found to be a perfectly acceptable substitute, giving the amidocyclopropanes in comparable yields (Entry 1). The formation of hard metal spheres during the course of the reactions is also no longer observed.
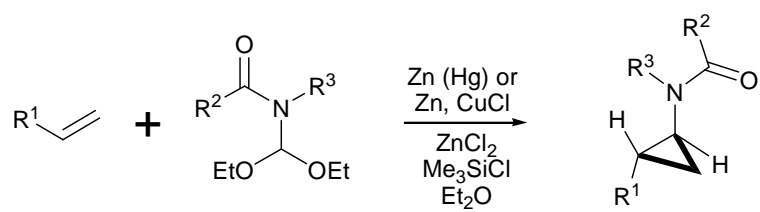

Scheme 3

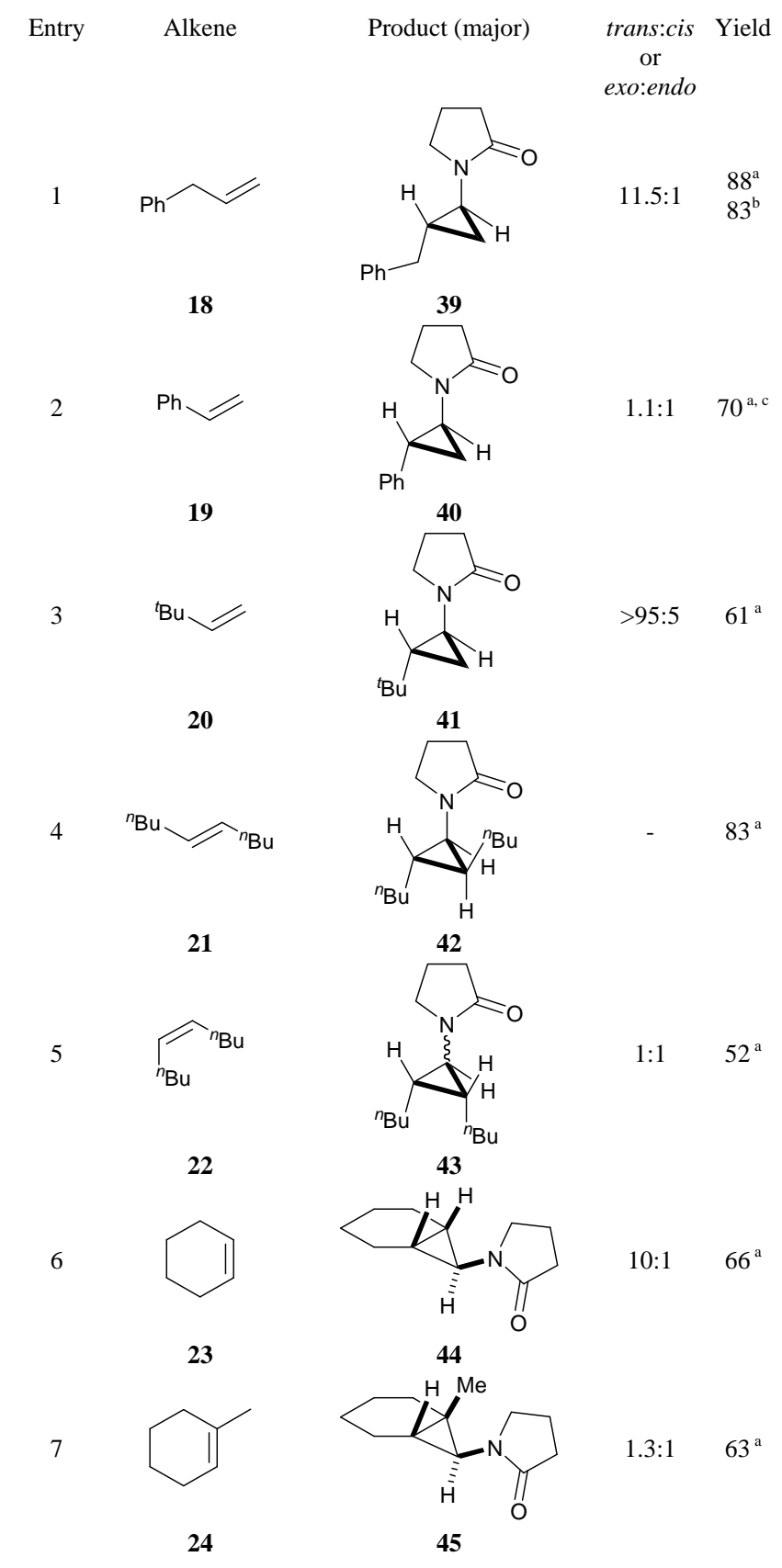


8<smiles>C=COC(=O)[C@H](CC)C(C)(C)C</smiles>

25<smiles>C=C(C)C(=O)OCC([13CH3])[18O]</smiles>

26

10<smiles>C=COC(=O)c1ccccc1</smiles>

27

$11 \quad \mathrm{Me}_{3} \mathrm{Si}$

28

12<smiles>C=C[AlH2]CCc1ccccc1</smiles>

13<smiles>C=CCOC(=O)c1ccccc1</smiles>

30

29<smiles>CCC(C)C(=O)OC1CC1N1CCCC1=O</smiles><smiles>C1CCCCC1</smiles><smiles>CCC(Br)COC(=O)C(C)(C)C1CC1</smiles>

$1: 1 \quad 9^{\mathrm{a}}$

$2: 1 \quad 56$<smiles>CC1CC1OC(=O)c1ccccc1</smiles><smiles></smiles>

49

14<smiles>C1=Cc2ccccc2C1</smiles>

31<smiles>O=C1C[C@@H]2[CH]CN12</smiles><smiles>C1CC2CC2C1</smiles>
$>95: 5 \quad 39^{\mathrm{a}}$<smiles>C1=Cc2ccccc2CC1</smiles>

32

16<smiles>C=CCOCC(=O)OCC(=O)OCC1C2C1C2N1CCCC1=O</smiles> 


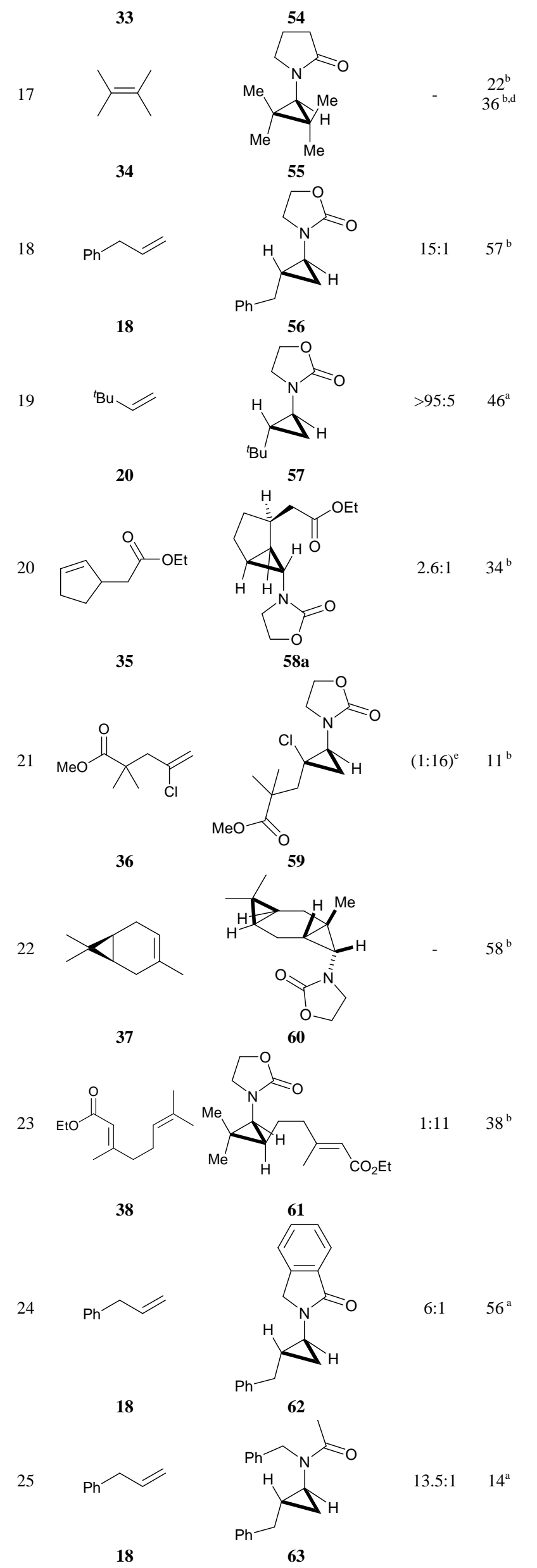




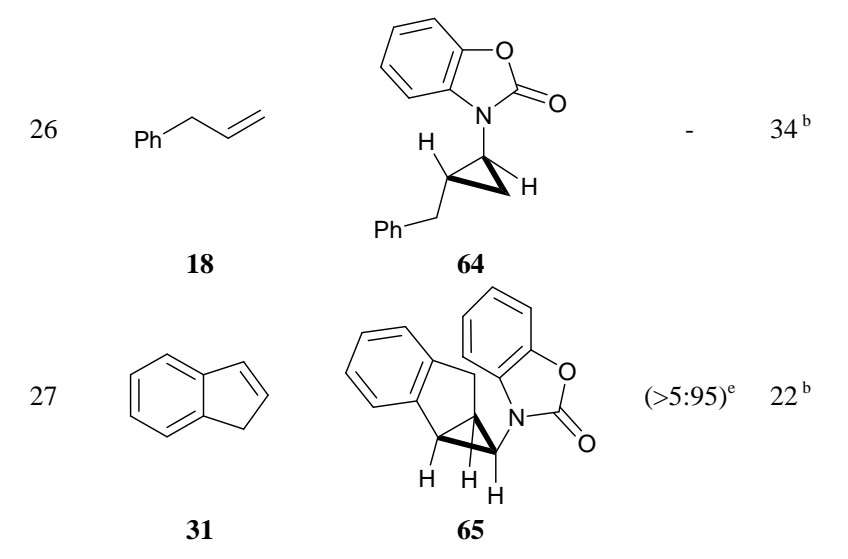

Table 2 a. $\mathrm{Zn}(\mathrm{Hg})$ method. b. $\mathrm{Zn} / \mathrm{CuCl}$ method. c. The carbenoid precursor was added by syringe pump over $2 \mathrm{~h}$. d. Reaction carried out at room temperature. e. Selectivities in parentheses refer to purified material.

The cyclopropane products 39-65 were obtained in moderate to good yields with a wide variety of alkenes. All of the carbenoid precursors could be employed in the amidocyclopropanation reaction with the exception of diethoxymethylphthalimide 14. Since the phthalimide functional group is known to be reduced under dissolving metal conditions, ${ }^{14}$ this observation is perhaps unsurprising. As anticipated, the amidocyclopropanation of electron-rich alkenes (Entries 8 and 10) proceeded in higher yield than the amidocyclopropanation of electron deficient alkenes (Entry 9) and in the cyclopropanation of diene 38 (Entry 23), chemoselective cyclopropanation of the more electron-rich alkene was observed. In terms of the steric environment, mono (Entries 1-3, 8, 10-13, 16, 18, 19, and 2426), di (Entries 4-6, 9, 14, 15, 20, 21, and 27), tri (Entries 7, 22, and 23) and tetrasubstituted (Entry 17) alkenes were all readily cyclpropanated under the reaction conditions and a variety of functional groups including esters, ethers, halides and silyl groups were tolerated. In the case of highly volatile alkenes (Entry 17), it was more effective to carry out the cyclopropanation reaction at room temperature. The stereochemistry of the major products was assigned on the basis of ${ }^{1} \mathrm{H}$ NMR coupling constants and simple proton decoupling experiments where required. In most cases, a preference for the formation of the less-hindered trans-amidocyclopropane was observed. However, the overall trends in selectivity from substrate to substrate are difficult to rationalise. In general, mono-substituted alkenes tend to favour the formation of the trans isomer with varying degrees of selectivity with larger substituents leading to a higher preference for trans-cyclopropanation. Interestingly, the presence of an allylic ether group reversed this selectivity (Entry 16), but an allylic or vinylic ester did not (Entries 8,10 and 13). This may indicate that coordination of the ether oxygen to the zinc carbenoid during cyclopropanation can influence the stereochemical outcome and we might expect this to be more favourable for an ether than an ester. The trends for more substituted alkenes are much more difficult to rationalise. Cyclohexene $\mathbf{2 3}$ showed a strong preference for the formation of the exo-cyclopropane (Entry 6), whereas for indene $\mathbf{3 1}$ the reverse was true (Entries 14 and 27). Apparently similar systems such as methylcyclohexene 24 and carene 37 showed contrasting selectivities in the cyclopropanation reaction (Entries 7 and 22). A speculative explanation using a quadrant model (Figure 1) in which one quadrant (A) is largely unhindered, but the adjacent quadrant $(\mathbf{B})$ is sterically congested might help to explain these results. Thus a monosubstituted alkene $\left(R^{2}=H\right)$ fits into the 'pocket' with the $R^{1}$ substituent occupying the unhindered quadrant $\mathbf{A}$ in transition state (i). When a second substituent is present on the alkene $\left(\mathrm{R}^{2} \neq \mathrm{H}\right)$, the larger steric hindrance in quadrant $\mathbf{B}$ can lead to the cis transition state (ii) becoming the favoured pathway, in which the two alkyl groups occupy quadrants $\mathbf{C}$ and $\mathbf{D}$ where there is only moderate steric hindrance.

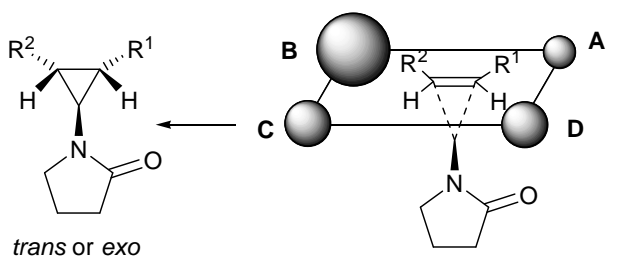

(i)

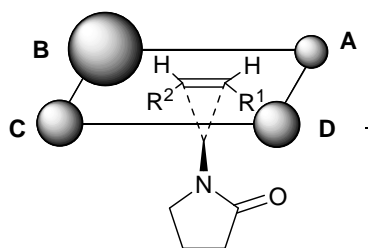

(ii)

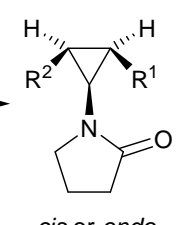

cis or endo 


\section{Figure 1}

A possible transition state in line with such a model is shown in Figure 2, in which the zinc atom is located in quadrant $\mathbf{D}$, and the orientation of the pyrrolidine ring is controlled by coordination of the lactam oxygen to the metal centre. This places the lactam carbonyl in quadrant $\mathbf{C}$ and the methylene adjacent to the nitrogen in quadrant $\mathbf{B}$, leaving quadrant $\mathbf{A}$ largely empty. Thus, a monosubstituted alkene $\left(\mathrm{R}^{2}=\mathrm{H}\right)$ can approach the carbenoid relatively easily with the $\mathrm{R}^{1}$ group located in quadrant $\mathbf{A}$, leading to a preference for trans-cyclopropane formation (i). With more substituted alkenes $\left(\mathrm{R}^{2} \neq \mathrm{H}\right)$, the unfavourable interaction between the lactam hydrogen atoms and $\mathrm{R}^{2}$ in the exo transition state (i) leads to a preference for the endo transition state (ii) in which $\mathrm{R}^{1}$ is close to the zinc atom and $\mathrm{R}^{2}$ is close to the lactam carbonyl group.

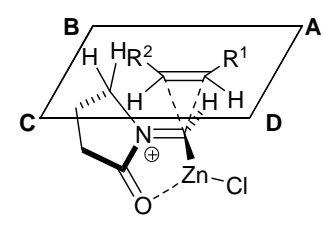

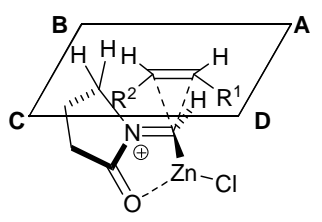

(ii)

Figure 2

The use of a large excess of chlorotrimethylsilane in the reaction precluded direct amidocyclopropanation of allylic alcohols, but a preliminary experiment (Scheme 4) with allyl benzene 18 demonstrated that the cyclopropane 39 could be produced in the absence of the silicon electrophile by employing a larger excess of zinc chloride (30\% conversion). When these alternative conditions were applied to the cyclopropanation of allyl alcohol, the amidocyclopropyl alcohol 67 was obtained in low yield (8\%, diastereomeric ratio trans:cis $2: 1$ ). However, when 2-cyclohexen-1-ol $\mathbf{6 8}$ was cyclopropanated under the same conditions, the only isolated product was the ethyl ether 69, again in $8 \%$ yield. Cyclopropane ether 69 is probably formed by initial displacement of the hydroxy group with ethanol under the Lewis acidic conditions and then subsequent cyclopropanation of the allylic ether 70. Alkene $\mathbf{7 0}$ was synthesised by alkylation of the alcohol 68, and cyclopropanated under the same conditions to give the same cyclopropane 69 in $20 \%$ yield (diastereomeric ratio >8:1). Interestingly, the ethyl ether appears to direct the approach of the organozinc carbenoid to the same face of the ring. ${ }^{15}$ The exo-cyclopropane is also formed selectively in a similar fashion to the cyclopropanation of cyclohexene above (Table 2, Entry 6).

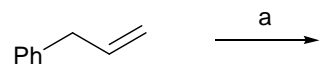

18<smiles>C=CCO</smiles>

66<smiles>OC1C=CCCC1</smiles>

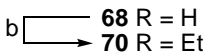

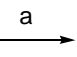<smiles>O=C1CCCN1C1CC1Cc1ccccc1</smiles><smiles>CC1CC1CO</smiles>

67

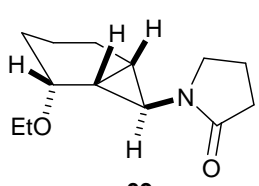

69 
Scheme 4 a. 12, $\mathrm{ZnCl}_{2}, \mathrm{Zn}, \mathrm{CuCl}, \mathrm{Et}_{2} \mathrm{O}, 8 \%$ (67), $8 \%$ (69 from 68), $20 \%$ (69 from 70). b. NaH, DMF, EtI, 33\% based on recovered $\mathbf{6 8}$.

The cyclopropanes formed in these reactions are suitable for further elaboration into derivatives of potential utility for incorporation as rigid conformational locks in a wide variety of systems, such as chiral ligands or drug molecules (Scheme 5). The oxazolidinone ring in cyclopropane 57 was opened under basic conditions to yield the aminoalcohol 71, which was BOC protected and oxidised to the corresponding $N$-cyclopropylglycine derivative 73. Similarly, the benzoxazolidine ring in 65 can be opened using Super Hydride ${ }^{16}$ to give the indenocyclopropylcatecholamine $\mathbf{7 4}$ in moderate yield. The cyclopropyl silane $\mathbf{5 0}$ was oxidised under Tamao-Fleming conditions to give the corresponding amidocyclopropanol 75 in $35 \%$ yield. These examples provide a brief illustration of the interesting cyclopropylamino acid and aminoalcohol units that can readily be prepared using amidoorganozinc carbenoids. Densely functionalised cyclopropylamino acid $\mathbf{7 3}$ was obtained in only four steps from the cheap commercially available alkene $\mathbf{2 0}$.

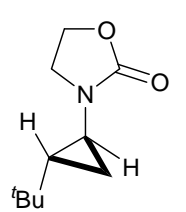

57<smiles>O=c1oc2ccccc2n1[C@H]1C[C@@H]2c3ccccc3[C@H]2[C@H]1C(F)(F)F</smiles>

65<smiles></smiles><smiles>CCCC</smiles>

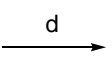

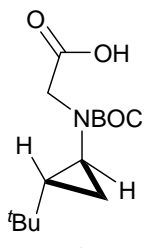

73<smiles>Oc1ccccc1N[C@@H]1C[C@@H]2c3ccccc3CC[C@@H]21</smiles>

74

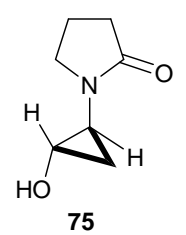

Scheme 5

a. $\mathrm{LiOH}, \mathrm{EtOH}, \mathrm{H}_{2} \mathrm{O}, 49 \%$. b. $\mathrm{BOC}_{2} \mathrm{O}, \mathrm{Et}_{3} \mathrm{~N}, \mathrm{CH}_{2} \mathrm{Cl}_{2}, 72 \%$. c. $\mathrm{KMnO}_{4}, \mathrm{NaOH},{ }^{t} \mathrm{BuOH}, \mathrm{H}_{2} \mathrm{O}, 84 \%$. d. $\mathrm{LiEt}_{3} \mathrm{BH}$, THF, 34\%. e. TBAF, THF, rt then $30 \% \mathrm{H}_{2} \mathrm{O}_{2}, \mathrm{KHCO}_{3}, \mathrm{MeOH}, 35 \%$.

Finally, from a practical viewpoint, it would be much more convenient to avoid the need for preparation of the orthoester derived carbenoid precursors. As both the introduction of the diethoxymethyl group itself and the cyclopropanation reaction are carried out in the presence of Lewis acids, we decided to investigate whether the two processes could be combined into a one-pot reaction (Scheme 6). Thus, triethyl orthoformate was added dropwise to a mixture of alkene 18, oxazolidinone 7, zinc, copper chloride, chlorotrimethylsilane and zinc chloride and to our delight, the amidocyclopropane was obtained directly in $33 \%$ yield, comparable to the $38 \%$ overall yield obtained in the separate steps described above. Although, the alkoxycyclopropanation of alkenes with orthoformates proceeds under similar conditions, ${ }^{10}$ no alkoxycyclopropane was observed, implying that the reaction between the amide and orthoester is overwhelmingly favoured. We are currently optimising this new approach and investigating its scope for the direct preparation of amidocyclopropanes from a wide range of amide precursors. 


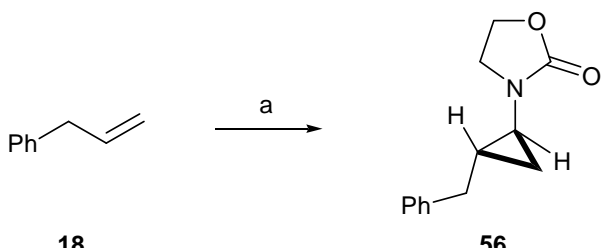

18

56

\section{Scheme 6}

a. 7, $(\mathrm{EtO})_{3} \mathrm{CH}, \mathrm{Me}_{3} \mathrm{SiCl}, \mathrm{ZnCl}_{2}, \mathrm{Zn}, \mathrm{CuCl}, \mathrm{Et}_{2} \mathrm{O}, 33 \%$.

In summary, the amidocyclopropanation of alkenes using dialkoxymethylamides as zinc carbenoid precursors constitutes an effective method for functionalised cyclopropane preparation, and enables a range of highly functionalised aminocyclopropane derivatives to be accessed, which cannot readily be obtained using existing methods. These amidocyclopropane units can be readily elaborated into cyclopropyl containing aminoalcohol or aminoacid motifs.

\section{Acknowledgments}

We would like to thank AstraZeneca for providing a studentship (to GB), and the EPSRC for providing both a studentship (to LJ) and a postdoctoral fellowship (to TDS). We would also like to thank Dr Abil Aliev for providing advice and assistance with NMR spectroscopy.

\section{Experimental}

All reactions were carried out in oven-dried glassware under a nitrogen atmosphere unless otherwise indicated. Diethyl ether, tetrahydrofuran, toluene and dichloromethane were used following purification from an anhydrous engineering zeolite drying apparatus. Methanol and ethanol were distilled from magnesium turnings and iodine. Anhydrous dimethylformamide was obtained from the Aldrich chemical company. Triethylamine was distilled from potassium hydroxide before use. Chlorotrimethylsilane was distilled from calcium hydride immediately prior to use. Styrene, cyclohexene, indene, 1,2-dihydronaphthalene and 3-carene were distilled before use. All other chemicals were used as supplied unless otherwise indicated. Column chromatography was carried out using BDH $(40-60 \mu \mathrm{m})$ silica gel and analytical thin layer chromatography was carried out using Merck Keiselgel aluminiumbacked plates coated with silica gel. Components were visualised using combinations of ultraviolet lights, iodine, ceric ammonium molybdate, phosphomolybdic acid and potassium permanganate. Melting points were determined using a Reichert hot-stage apparatus and are uncorrected. Optical rotations were measured using a Perkin-Elmer 241 (sodium D-line, 529 $\mathrm{nm})$ polarimeter and $[\alpha]$ values are given in $10^{-1} \mathrm{deg} \mathrm{cm}^{2} \mathrm{~g}^{-1}$, concentration $(c)$ in $\mathrm{g}$ per 100 $\mathrm{mL}$. Infrared (IR) spectra were recorded on a Perkin-Elmer 1605 Fourier transform spectrometer, and were recorded as thin films ( $\mathrm{NaCl}$ plate). ${ }^{1} \mathrm{H}$ NMR spectra were recorded at $300 \mathrm{MHz}$ on a Bruker AMX300 spectrometer, at $400 \mathrm{MHz}$ on a Bruker AMX400 spectrometer or at $500 \mathrm{MHz}$ on a Bruker Avance 500 spectrometer in the stated solvent using residual protic solvent $\mathrm{CHCl}_{3}(\delta=7.26 \mathrm{ppm}, \mathrm{s})$, DMSO $\left(\delta=2.56 \mathrm{ppm}\right.$, qn) or $\mathrm{D}_{2} \mathrm{O}(4.79, \mathrm{~s})$ as the internal standard. Chemical shifts are quoted in ppm using the following abbreviations: s, singlet; d, doublet; $t$, triplet; q, quartet; qn, quintet; m, multiplet; br, broad or a combination of these. The coupling constants $(J)$ are measured in Hertz. ${ }^{13} \mathrm{C}$ NMR spectra were recorded at $75 \mathrm{MHz}$ on a Bruker AMX300 spectrometer, at $100 \mathrm{MHz}$ on a Bruker AMX400 spectrometer or at $125 \mathrm{MHz}$ on a Bruker Avance 500 spectrometer in the stated solvent using the central reference of $\mathrm{CHCl}_{3}(\delta=77.0 \mathrm{ppm}, \mathrm{t})$, DMSO $(\delta=39.52 \mathrm{ppm}$, septet $)$ as the internal standard. Chemical shifts are reported to the nearest $0.1 \mathrm{ppm}$. Mass spectra and elemental analysis were performed at the Department of Chemistry, University College London.

\section{2,3-Dihydro-1-isoindolinone $9^{14}$}

Tin powder ( $41.7 \mathrm{~g}, 0.35 \mathrm{~mol}, 2.6 \mathrm{eq})$ was added to a vigorously stirred suspension of phthalimide $(20 \mathrm{~g}, 0.14 \mathrm{~mol}, 1 \mathrm{eq})$ in a mixture of glacial acetic acid $(100 \mathrm{~mL})$ and concentrated hydrochloric acid $(50 \mathrm{~mL})$. The reaction mixture was heated at reflux for $2 \mathrm{~h}$ and then filtered hot. The filtrate was concentrated and the residue partitioned between dichloromethane $(300 \mathrm{~mL})$ and water $(150 \mathrm{~mL})$. The organic layer was separated and the aqueous layer extracted with dichloromethane $(2 \times 50 \mathrm{~mL})$. The combined organic extracts were dried $\left(\mathrm{MgSO}_{4}\right)$, filtered and concentrated. The crude product was purified twice by flash column chromatography (EtOAc) and then triturated in toluene to give the lactam 9 (4.2 g, 
$31.5 \mathrm{mmol}, 23 \%)$ as a white solid; $\mathrm{Mp} 149-150{ }^{\circ} \mathrm{C}$ (lit. $\left.150-151{ }^{\circ} \mathrm{C}^{14}\right)$; IR $\left(\mathrm{CH}_{2} \mathrm{Cl}_{2}\right): v_{\max } 3214$ (br s), 1658 (s) cm ${ }^{-1}$; $\mathrm{H}$ NMR (400 MHz, $\mathrm{CDCl}_{3}$ ): $\delta 4.48$ (s, 2H, $\mathrm{CH}_{2}$ ), $7.18($ br s, $1 \mathrm{H}, \mathrm{NH}$ ), 7.47-7.60 (m, $4 \mathrm{H}, \mathrm{Ar}-H) ;{ }^{13} \mathrm{C}$ NMR $\left(100 \mathrm{MHz}, \mathrm{CDCl}_{3}\right): \delta 45.8,123.2,123.6,128.0,131.7$, 132.6, 143.7, 172.6.

\section{General procedure for synthesis of diethoxymethylamides 12-17}

A mixture of amide $(6.7 \mathrm{mmol})$, aluminium chloride $(1.0 \mathrm{mmol}, 0.15 \mathrm{eq})$ and triethyl orthoformate $(22 \mathrm{~mL}, 0.13 \mathrm{~mol}, 20 \mathrm{eq})$ was heated at $155^{\circ} \mathrm{C}$ for $24-72 \mathrm{~h}$. The reaction mixture was allowed to cool to room temperature and then quenched with saturated aqueous sodium bicarbonate $(25 \mathrm{~mL})$. The aqueous phase was extracted with diethyl ether (50 mL then $2 \times 25$ $\mathrm{mL})$ and the combined organic extracts were washed with brine $(25 \mathrm{~mL})$, dried $\left(\mathrm{Na}_{2} \mathrm{SO}_{4}\right)$, filtered and concentrated. The crude product was purified by flash column chromatography.

\section{$N$-Diethoxymethylpyrrolidin-2-one 12 (alternative procedure)}

Chlorotrimethylsilane $(1.5 \mathrm{~mL}, 11.7 \mathrm{mmol})$ was added dropwise to a stirred solution of pyrrolidinone $(10 \mathrm{~g}, 117.6 \mathrm{mmol})$, triethyl orthoformate $(50 \mathrm{~mL}, 300.6 \mathrm{mmol})$ and triphenylphosphine $(50 \mathrm{mg}, 0.2 \mathrm{mmol})$ in dichloromethane $(200 \mathrm{~mL})$ under nitrogen. The resulting solution was stirred overnight at room temperature then poured into water $(70 \mathrm{~mL})$ and saturated sodium bicarbonate $(50 \mathrm{~mL})$. The layers were separated and the organic layer washed with brine $(50 \mathrm{~mL})$, dried $\left(\mathrm{Na}_{2} \mathrm{SO}_{4}\right)$ and concentrated. The residue was distilled under reduced pressure to give 12 as a colourless liquid (bp $101-103{ }^{\circ} \mathrm{C}$ at 3 mbar [lit. $84-86{ }^{\circ} \mathrm{C}$ at 1 $\left.\left.\mathrm{mmHg}^{17}\right], 13.42 \mathrm{~g}, 61 \%\right)$.

\section{$N$-Diethoxymethyl-2-oxazolidinone 13 (alternative procedure)}

Acetyl chloride ( $1 \mathrm{~mL}, 14 \mathrm{mmol})$ was added to a stirred suspension of oxazolidin-2-one (10 g, $113.8 \mathrm{mmol})$ in triethyl orthoformate $(50 \mathrm{~mL}, 300.6 \mathrm{mmol})$. The suspension was stirred overnight at room temperature and then the reaction mixture was distilled under reduced pressure to give diethoxymethyloxazoldinone as a colourless liquid $\left(120-122{ }^{\circ} \mathrm{C}\right.$ at 0.9 $\mathrm{mmHg}, 12.9 \mathrm{~g}, 60 \%)$.

\section{$N$-Diethoxymethylpyrrolidin-2-one 12}

Pale yellow oil; IR (film): $v_{\max } 2977$ (s), 2897 (m), 1693 (s) $\mathrm{cm}^{-1} ;{ }^{1} \mathrm{H}$ NMR (300 MHz, $\left.\mathrm{CDCl}_{3}\right): \delta 1.20(\mathrm{t}, J=7.0,6 \mathrm{H}, 2 \times \mathrm{Me}), 1.96-2.03\left(\mathrm{~m}, 2 \mathrm{H}, \mathrm{CH}_{2} \mathrm{CH}_{2} \mathrm{~N}\right), 2.41(\mathrm{t}, J=8.2,2 \mathrm{H}$, $\left.\mathrm{CH}_{2} \mathrm{CON}\right), 3.42\left(\mathrm{t}, J=7.3, \mathrm{CH}_{2} \mathrm{~N}\right), 3.48(\mathrm{dq}, J=9.4,7.0,2 \mathrm{H}, 2 \times \mathrm{CH} \mathrm{HMe}), 3.62(\mathrm{dq}, J=9.4$, $7.0,2 \mathrm{H}, 2 \times \mathrm{CH} H \mathrm{Me}), 5.87(\mathrm{~s}, 1 \mathrm{H}, \mathrm{CH}) ;{ }^{3} \mathrm{C} \mathrm{NMR}\left(75 \mathrm{MHz}, \mathrm{CDCl}_{3}\right): \delta 14.5,17.8,31.4,40.5$, 61.9, 98.8, 175.2; MS (FAB) m/z (\%): $210(\mathrm{M}+\mathrm{Na}, 100), 142$ (25); HMRS: M+Na, found 210.11009. $\mathrm{C}_{9} \mathrm{H}_{17} \mathrm{NO}_{3} \mathrm{Na}$ requires 210.11061 .

\section{$N$-Diethoxymethyl-2-oxazolidinone 13}

Pale yellow oil; IR (film): $v_{\max } 2979$ (w), 2904 (w), 1747 (s) $\mathrm{cm}^{-1} ;{ }^{1} \mathrm{H}$ NMR (400 MHz, $\left.\mathrm{CDCl}_{3}\right): \delta 1.25(\mathrm{t}, J=7.1,6 \mathrm{H}, \mathrm{Me}), 3.53-3.73\left(\mathrm{~m}, 6 \mathrm{H}, \mathrm{CH}_{2} \mathrm{~N}\right.$ and $\left.2 \times \mathrm{CH}_{2} \mathrm{Me}\right), 4.39(\mathrm{t}, J=8.2$, $\left.2 \mathrm{H}, \mathrm{CH}_{2} \mathrm{CH}_{2} \mathrm{~N}\right), 5.74(\mathrm{~s}, 1 \mathrm{H}, \mathrm{CH}) ;{ }^{3} \mathrm{C} \mathrm{NMR}\left(100 \mathrm{MHz}, \mathrm{CDCl}_{3}\right): \delta 14.8,38.6,62.6,62.8$, 101.3, 157.4; MS (CI+) m/z (\%): 190 (M+H, 2), 144, 100), 116 (12), 103 (100), 75 (14), 44 (16); HMRS: $\mathrm{M}+\mathrm{H}$, found 190.10785. $\mathrm{C}_{8} \mathrm{H}_{16} \mathrm{NO}_{4}$ requires 190.10793.

\section{$N$-Diethoxymethylphthalimide 14}

White solid; Mp 73-74 ${ }^{\circ} \mathrm{C}$ (lit., $73{ }^{\circ} \mathrm{C}^{18}$ ); IR (film): $v_{\max } 2977$ (w), 1774 (m), 1720 (s), 1694 (w), $1469(\mathrm{w}) \mathrm{cm}^{-1} ;{ }^{1} \mathrm{H}$ NMR $\left(300 \mathrm{MHz}, \mathrm{CDCl}_{3}\right): \delta 1.25\left(\mathrm{t}, J=7.1,6 \mathrm{H}, 2 \mathrm{x} \mathrm{CH}_{3}\right), 3.62(\mathrm{dq}$, $J=9.4,7.1,2 \mathrm{H}, 2 \times \mathrm{CH} \mathrm{HMe}), 3.77(\mathrm{dq}, J=9.4,7.1,2 \mathrm{H}, 2 \times \mathrm{CH} H \mathrm{Me}), 6.09(\mathrm{~s}, 1 \mathrm{H}, \mathrm{CH}), 7.68-$ $7.77(\mathrm{~m}, 2 \mathrm{H}, \mathrm{Ar}-\mathrm{H}), 7.82-7.90(\mathrm{~m}, 2 \mathrm{H}, \mathrm{Ar}-\mathrm{H}) ;{ }^{13} \mathrm{C} \mathrm{NMR}\left(75 \mathrm{MHz}, \mathrm{CDCl}_{3}\right): \delta 14.8,63.4,99.8$, 123.6, 131.7, 134.3, 166.6; MS (CI+) m/z (\%): 249 (M+1 100), 175 (56), 147 (78); HMRS: $\mathbf{M}^{+}$, found 249.09952. $\mathrm{C}_{13} \mathrm{H}_{15} \mathrm{NO}_{4}$ requires 249.1001.

\section{2-Diethoxymethyl-2,3-dihydro-1-isoindolinone 15}

Brown oil; IR (film): $v_{\max } 2977$ (m), 2932 (w), 1694 (s), 1620 (w), 1470 (m), 1452 (w) cm cm $^{-1}$; ${ }^{1} \mathrm{H}$ NMR $\left(400 \mathrm{MHz}, \mathrm{CDCl}_{3}\right): \delta 1.25(\mathrm{t}, J=7.0,6 \mathrm{H}, 2 \times \mathrm{Me}), 3.57(\mathrm{dq}, J=9.5,7.0,2 \mathrm{H}, 2 \times$ $\left.\mathrm{CHHCH}_{3}\right), 3.74\left(\mathrm{dq}, J=9.5,7.0,2 \mathrm{H}, 2 \times \mathrm{CHHCH}_{3}\right), 4.49\left(\mathrm{~s}, 2 \mathrm{H}, \mathrm{CH}_{2} \mathrm{~N}\right), 6.23(\mathrm{~s}, 1 \mathrm{H}, \mathrm{CH})$, 7.45-7.51 (m, $2 \mathrm{H}, \mathrm{Ar}-H), 7.58(\mathrm{t}, J=7.5,1 \mathrm{H}, \mathrm{Ar}-H), 7.87(\mathrm{~d}, J=7.5,1 \mathrm{H}, \mathrm{Ar}-H) ;{ }^{3} \mathrm{C}$ NMR $(100$ 
$\left.\mathrm{MHz}, \mathrm{CDCl}_{3}\right): \delta 14.8,44.2,62.5,99.2,123.2,124.1,128.0,132.0,132.1,142.0,168.9$; MS (CI+) $m / z(\%): 236(\mathrm{M}+\mathrm{H}, 6), 190$ (44), 134 (59), 103 (46); HMRS: M+H, found 236.12847. $\mathrm{C}_{13} \mathrm{H}_{18} \mathrm{NO}_{3}$ requires 236.12866 .

\section{3-(Diethoxymethyl)benzoxazolin-2-one 16}

Red oil; IR (film): $v_{\max }$ 2980, 1786 (s), 1625 (w); ${ }^{1} \mathrm{H}$ NMR (400 MHz, DMSO): $\delta 1.16$ (t, $J=7.1,6 \mathrm{H}, 2 \times \mathrm{Me}), 3.60(\mathrm{dq}, J=9.6,7.1,2 \mathrm{H}, 2 \times \mathrm{CHHMe}), 3.73(\mathrm{dq}, J=9.6,7.1,2 \mathrm{H}, 2 \times$ $\mathrm{CH} H \mathrm{Me}), 6.11(\mathrm{~s}, 1 \mathrm{H}, \mathrm{CH}), 7.16-7.24(\mathrm{~m}, 2 \mathrm{H}, \mathrm{Ar}), 7.34-7.39(\mathrm{~m}, 2 \mathrm{H}, \mathrm{Ar}) ;{ }^{13} \mathrm{C} \mathrm{NMR}:(100$ MHz, DMSO) 14.6, 62.4, 101.4, 110.0, 111.9, 122.9, 124.2, 127.7, 141.9, 152.4. MS (CI+) $\mathrm{m} / \mathrm{z}$ (\%): $237\left(\mathrm{M}^{+}, 70\right), 205$ (75), 193 (100), 104 (70); HRMS: M+H, found: 238.10832. $\mathrm{C}_{12} \mathrm{H}_{16} \mathrm{NO}_{4}$ requires 238.10793 .

\section{$N$-Benzyl- $N$-diethoxymethylacetamide 17}

Yellow oil; IR (film): $v_{\max } 2978$ (m), 2933 (w), 1670 (s), 1497 (w) $\mathrm{cm}^{-1} ;{ }^{1} \mathrm{H}$ NMR (400 MHz, $353 \mathrm{~K}, \mathrm{DMSO}): \delta 1.10(\mathrm{t}, J=7.1,6 \mathrm{H}, 2 \times \mathrm{Me}), 2.05\left(\mathrm{~s}, 2.1 \mathrm{H}, \mathrm{COCH}_{3}\right), 2.98(\mathrm{~s}, 0.9 \mathrm{H}$, $\left.\mathrm{COCH}_{3}\right), 3.37-3.63\left(\mathrm{~m}, 4 \mathrm{H}, 2 \times \mathrm{CH}_{2} \mathrm{CH}_{3}\right), 4.41\left(\mathrm{~s}, 0.6 \mathrm{H}, \mathrm{PhCH}_{2} \mathrm{~N}\right), 4.50\left(\mathrm{~s}, 1.4 \mathrm{H}, \mathrm{PhCH}_{2} \mathrm{~N}\right)$, 5.58 (br s, $0.3 \mathrm{H}, \mathrm{CH}), 6.00$ (br s, 0.7H, $\mathrm{CH}$ ), 7.16-7.35 (m, 5H, Ar- $H$ ); ${ }^{13} \mathrm{C}$ NMR (major rotamer, $100 \mathrm{MHz}, 353 \mathrm{~K}, \mathrm{DMSO}): \delta 14.0,21.4,43.7,61.2,102.2,125.8,126.7,127.3$, 138.9, 169.0; MS (CI+) m/z (\%): 252 (M+H, 3), 206 (62), 150 (100), 103 (61), 91 (68); HMRS: $\mathrm{M}+\mathrm{H}$, found 252.16019. $\mathrm{C}_{14} \mathrm{H}_{22} \mathrm{NO}_{3}$ requires 252.15997.

\section{Benzyldimethyl(vinyl)silane 29}

A solution of benzyl chloride $(1.15 \mathrm{~mL}, 10 \mathrm{mmol})$ in dry diethyl ether $(10 \mathrm{~mL})$ was added dropwise to a mixture of magnesium turnings $(0.24 \mathrm{~g}, 10 \mathrm{mmol})$ and iodine (2 crystals) in dry diethyl ether $(10 \mathrm{~mL})$ under nitrogen. The reaction mixture was stirred for $1 \mathrm{~h}$ and dimethylvinylchlorosilane $(1.64 \mathrm{~mL}, 12 \mathrm{mmol})$ was added dropwise. The reaction mixture was heated at reflux for $16 \mathrm{~h}$ and then quenched carefully with saturated aqueous ammonium chloride $(20 \mathrm{~mL})$. The organic layer was separated and the aqueous layer was extracted with diethyl ether $(2 \times 20 \mathrm{~mL})$. The combined organic extracts were dried $\left(\mathrm{MgSO}_{4}\right)$, filtered and concentrated in vacuo. The crude product was purified by flash column chromatography (Petrol) to give 29 as a colourless oil $(1.09 \mathrm{~g}, 62 \%)$; IR $\left(\mathrm{CDCl}_{3}\right): v_{\max } 3024,2957,2893(\mathrm{w})$, 1601, 1493 (s), 1452 (w), $1404 \mathrm{~cm}^{-1}$; ${ }^{1} \mathrm{H}$ NMR (500 MHz, CDCl 3 ): $\delta 0.57(\mathrm{~s}, 6 \mathrm{H}, 2 \times \mathrm{Me})$, $2.15\left(\mathrm{~s}, 2 \mathrm{H}, \mathrm{CH}_{2}\right), 5.67(\mathrm{dd}, J=20.3,3.8,1 \mathrm{H}, \mathrm{CH}=\mathrm{CH}), 5.97(\mathrm{dd}, J=14.7,3.8,1 \mathrm{H}$, $\mathrm{CH}=\mathrm{CH} H), 6.13\left(\mathrm{dd}, J=20.3,14.7,1 \mathrm{H}, \mathrm{CH}=\mathrm{CH}_{2}\right), 6.99-7.02(\mathrm{~m}, 2 \mathrm{H}, \mathrm{Ar}-H), 7.05-7.09(\mathrm{~m}$, $1 \mathrm{H}, \operatorname{Ar}-H), 7.18-7.23(\mathrm{~m}, 2 \mathrm{H}, \mathrm{Ar}-H) ;{ }^{13} \mathrm{C}$ NMR $\left(125 \mathrm{MHz}, \mathrm{CDCl}_{3}\right): \delta-3.7,25.8,124.0$, 128.1, 128.2, 132.2, 138.2, 139.9; MS (EI) m/z (\%): 176 (M+, 13), 91 (17), 85 (100); HMRS: $\mathrm{M}^{+}$, found 176.10155. $\mathrm{C}_{11} \mathrm{H}_{16} \mathrm{Si}$ requires 176.10158.

\section{Ethyl 2-allyloxyacetate $33^{19}$}

Sodium hydride (60\% dispersion in mineral oil, $1.76 \mathrm{~g}, 44 \mathrm{mmol}$ ) was added portionwise to a solution of ethyl glycolate $(4.16 \mathrm{~g}, 40 \mathrm{mmol})$ in anhydrous DMF $(20 \mathrm{~mL})$ under nitrogen at 0 ${ }^{\circ} \mathrm{C}$. After stirring for 30 minutes, allyl bromide $(3.8 \mathrm{~mL}, 44 \mathrm{mmol})$ was added dropwise and the reaction mixture was allowed to warm to room temperature. After stirring for $90 \mathrm{~min}$ at room temperature, water $(20 \mathrm{~mL})$ was added and the reaction mixture extracted with ether (3 $\times 30 \mathrm{~mL})$. The combined organic layers were washed with brine, dried $\left(\mathrm{MgSO}_{4}\right)$ and concentrated and the residue was distilled to afford two fractions: Fraction 1 (bp 52-54 ${ }^{\circ} \mathrm{C}, 14$ mbar) contained a mixture of the alkene 33 and DMF (2:1 ratio), Fraction 2 (bp 64-65 ${ }^{\circ} \mathrm{C}, 10$ mbar, lit. $70-74{ }^{\circ} \mathrm{C}$ at $20 \mathrm{mmHg}^{19}$ ) contained the alkene 33. The two fractions were combined and dissolved in ether $(30 \mathrm{~mL})$ and washed with saturated lithium chloride $(2 \times 20 \mathrm{~mL})$, dried $\left(\mathrm{MgSO}_{4}\right)$, and concentrated to give the alkene as a colourless liquid $(1.37 \mathrm{~g}, 9.5 \mathrm{mmol}, 24 \%)$; IR (film): $v_{\max } 2984,2941,2909,2876,1755$ (s) cm ${ }^{-1} ;{ }^{1} \mathrm{H}$ NMR $\left(500 \mathrm{MHz}, \mathrm{CDCl}_{3}\right): \delta 1.29(\mathrm{t}$, $J=7.2,3 \mathrm{H}, \mathrm{Me}), 4.07\left(\mathrm{~s}, 2 \mathrm{H}, \mathrm{CH}_{2} \mathrm{O}\right), 4.10\left(\mathrm{dt}, J=5.8,1.5,2 \mathrm{H}, \mathrm{CH}_{2} \mathrm{CH}\right), 4.22(\mathrm{q}, J=7.2$, $\mathrm{CH}_{2} \mathrm{Me}$ ), $5.26(\mathrm{dq}, J=10.3,1.5,1 \mathrm{H}, \mathrm{CH}=\mathrm{CH}), 5.33(\mathrm{dd}, J=17.1,1.5, \mathrm{CH} H=\mathrm{CH}), 5.93$ (ddt, $J=17.1,10.3,5.8, \mathrm{CH}=\mathrm{CHH}) ;{ }^{13} \mathrm{C}$ NMR $\left(100 \mathrm{MHz} \mathrm{CDCl}_{3}\right): \delta 14.1,60.8,67.1,72.3,118.2$, 133.6, 170.3 .

\section{Ethyl 2-(cyclopent-2-enyl)acetate $35^{20}$}

Acetyl chloride ( $3 \mathrm{~mL}, 42.4 \mathrm{mmol})$ was added dropwise to a solution of ethanol $(30 \mathrm{~mL})$ at 0 ${ }^{\circ} \mathrm{C}$. 2-(Cyclopent-2-enyl)acetic acid $(2 \mathrm{~g}, 15.9 \mathrm{mmol})$ was added dropwise to the solution at 0 ${ }^{\circ} \mathrm{C}$. The resulting mixture was allowed to warm to room temperature and was then heated to 
reflux for 4 hours. After concentration under reduced pressure the residue was dissolved in chloroform and concentrated under reduced pressure again to give a biphasic mixture. The mixture was extracted with dichloromethane $(10 \mathrm{~mL})$ and the combined organic layers were dried over $\mathrm{MgSO}_{4}$ and concentrated under reduced pressure. The crude product was purified by flash chromatography (petrol:ether, 4:1) to give the ester as a pale yellow oil (1.6 g, $65 \%)$; IR (film): $v_{\max } 2907(\mathrm{~s}), 1732(\mathrm{~s}), 1614(\mathrm{w}), 1259(\mathrm{~s}), 1148(\mathrm{~s}), 1032(\mathrm{~s}) \mathrm{cm}^{-1} ;{ }^{1} \mathrm{H}$ NMR (500 $\left.\mathrm{MHz}, \mathrm{CDCl}_{3}\right): \delta 1.26\left(\mathrm{t}, J=7.2,3 \mathrm{H}, \mathrm{CH}_{3}\right), 1.42-1.50\left(\mathrm{~m}, 1 \mathrm{H}, \mathrm{CH} \mathrm{HCHCH}_{2}\right), 2.07-2.16(\mathrm{~m}, 1 \mathrm{H}$, $\left.\mathrm{CH} \mathrm{CHCH}_{2}\right), 2.25-2.33\left(\mathrm{~m}, 2 \mathrm{H}, \mathrm{CH}_{2} \mathrm{C}=\mathrm{O}\right), 2.33-2.42\left(\mathrm{~m}, 2 \mathrm{H}, \mathrm{CH}_{2} \mathrm{CH}=\mathrm{CH}\right), 3.04-3.12(\mathrm{~m}$, $1 \mathrm{H}, \mathrm{CHCH}=\mathrm{CH}), 4.14\left(\mathrm{q}, J=7.2,2 \mathrm{H}, \mathrm{CH}_{2} \mathrm{CH}_{3}\right), 5.65-5.68(\mathrm{~m}, 1 \mathrm{H}, \mathrm{CH}=\mathrm{CH}), 5.74-5.78(\mathrm{~m}$, $1 \mathrm{H}, \mathrm{CH}=\mathrm{CH}) ;{ }^{13} \mathrm{C} \mathrm{NMR}\left(125 \mathrm{MHz}, \mathrm{CDCl}_{3}\right): \delta 14.3,29.6,31.8,40.5,41.8,60.2,131.4,133.7$, 173.0; MS (EI) $\mathrm{m} / z$ (\%): 153 (M-H, 33), 79 (75), 57 (100); HMRS: M-H, found 153.09115. $\mathrm{C}_{9} \mathrm{H}_{13} \mathrm{O}_{2}$ requires 153.09101 .

\section{(E)-Ethyl 3,7-dimethylocta-2,6-dienoate 38}

Sodium hydride (60\% mineral oil dispersion, $0.312 \mathrm{~g}, 7.80 \mathrm{mmol}$ ) was washed three times with hexane under nitrogen. The resulting solid was suspended in dry THF (10 mL) cooled to $0{ }^{\circ} \mathrm{C}$. A solution of triethyl phosphonoacetate $(1.75 \mathrm{~g}, 7.8 \mathrm{mmol})$ in tetrahydrofuran $(6 \mathrm{~mL})$ was added with stirring at $0{ }^{\circ} \mathrm{C}$, and the mixture was stirred at this temperature for a further 30 min. A solution of 6-methyl-5-hepten-2-one $(0.74 \mathrm{~g}, 5.9 \mathrm{mmol})$ in tetrahydrofuran $(5 \mathrm{~mL})$ was added and the resulting mixture was stirred at $0{ }^{\circ} \mathrm{C}$ for 1 hour and then allowed to warm to room temperature and stirred for 16 hours. The residue was then poured into a mixture of ether $(50 \mathrm{~mL})$ and a saturated aqueous ammonium chloride $(20 \mathrm{~mL})$. The ether layer was separated and the aqueous layer extracted with ether $(2 \times 20 \mathrm{~mL})$. The combined organic layers were dried over $\mathrm{MgSO}_{4}$ and concentrated under reduced pressure. The crude product (approximately 75:25 E:Z) was purified by column chromatography ( $3 \%{ }^{t} \mathrm{BuOMe}$ in hexane) to give $1.03 \mathrm{~g}$ of a mixture of $(E)$ and $(Z)$ isomers as a colourless oil and $187 \mathrm{mg}$ of the pure $E$ isomer as a colourless oil ( $89 \%$ overall yield). The pure $E$ isomer was used in the cyclopropanation reaction; IR (film): $v_{\max } 1643(\mathrm{~s}), 1385$ (w), $1223(\mathrm{w}), 1142 \mathrm{~cm}^{-1} ;{ }^{1} \mathrm{H}$ NMR $\left(400 \mathrm{MHz}, \mathrm{CDCl}_{3}\right): \delta 1.28\left(\mathrm{t}, J=7.1,3 \mathrm{H}, \mathrm{CH}_{3} \mathrm{CH}_{2}\right), 1.60\left(\mathrm{~s}, 3 \mathrm{H}, \mathrm{CH}_{3} \mathrm{CCH}_{3}\right), 1.68(\mathrm{~s}, 3 \mathrm{H}$, $\left.\mathrm{CH}_{3} \mathrm{CCH}_{3}\right) 2.15\left(\mathrm{~s}, 3 \mathrm{H}, \mathrm{CH}_{3} \mathrm{C}=\mathrm{CH}\right), 2.16\left(\mathrm{~m}, 4 \mathrm{H}, 2 \times \mathrm{CH}_{2}\right), 4.15\left(\mathrm{q}, \mathrm{J}=7.1,2 \mathrm{H}, \mathrm{CH}_{2} \mathrm{CH}_{3}\right)$, $5.03-5.1(\mathrm{~m}, 1 \mathrm{H}, \mathrm{CHCOO}), 5.66\left(\mathrm{br} \mathrm{s}, 1 \mathrm{H}, \mathrm{Me}_{2} \mathrm{C}=\mathrm{CH}\right) ;{ }^{13} \mathrm{C}$ NMR $\left(125 \mathrm{MHz}, \mathrm{CDCl}_{3}\right): \delta 14.3$, 17.7, 18.8, 25.7, 26.0, 40.9, 59.5, 115.6, 123.0, 132.5, 159.8, 166.9; MS (EI) m/z (\%): 196 $\left(\mathrm{M}^{+}, 10\right), 151$ (33), 123 (60), 97 (52), 83 (100); HRMS: $\mathrm{M}^{+}$, found 196.14572. $\mathrm{C}_{12} \mathrm{H}_{20} \mathrm{O}_{2}$ requires 196.14632 .

\section{Preparation of Zinc amalgam}

Zinc dust (10.0 g, $153 \mathrm{mmol})$ was added portionwise to a vigorously stirred solution of mercury (II) chloride $(2.0 \mathrm{~g}, 7.20 \mathrm{mmol})$ in concentrated hydrochloric acid $(2 \mathrm{~mL})$ and water $(30 \mathrm{~mL})$. The suspension was stirred for $10 \mathrm{~min}$ and then filtered and the zinc amalgam washed with water $(3 \times 20 \mathrm{~mL})$, acetone $(3 \times 20 \mathrm{~mL})$, ethanol $(3 \times 20 \mathrm{~mL})$ and ether $(3 \times 20$ $\mathrm{mL}$ ) and then dried under vacuum.

\section{Zinc dust for cyclopropanation reactions}

A suspension of commerically available zinc dust $(5 \mathrm{~g}, 76.5 \mathrm{mmol})$ in $2 \%$ hydrochloric acid $(15 \mathrm{~mL})$ was stirred for $10 \mathrm{~min}$ and then filtered and the collected zinc dust washed with water $(3 \times 20 \mathrm{~mL})$, acetone $(3 \times 20 \mathrm{~mL})$, ethanol $(3 \times 20 \mathrm{~mL})$ and ether $(3 \times 20 \mathrm{~mL})$ and then dried under vacuum.

\section{General procedure for the cyclopropanation of alkenes using Zinc amalgam}

A solution of diethoxymethylamide $(2.11 \mathrm{mmol})$ in dry diethyl ether $(3 \mathrm{~mL})$ was added via a motorised syringe pump over 4-6 h to a vigorously stirred mixture of zinc amalgam (1.38 g, $21.1 \mathrm{mmol})$, zinc chloride $(1.0 \mathrm{M}$ solution in diethyl ether, $2.11 \mathrm{~mL}, 2.11 \mathrm{mmol})$, chlorotrimethylsilane $(1.34 \mathrm{~mL}, 10.56 \mathrm{mmol})$ and alkene $(1.06 \mathrm{mmol})$ in dry diethyl ether $(5.5$ $\mathrm{mL}$ ) under nitrogen at reflux. The mixture was stirred at reflux overnight and then allowed to cool to room temperature. The reaction was quenched with saturated aqueous sodium bicarbonate solution $(20 \mathrm{~mL})$ and after stirring for $20 \mathrm{~min}$, the mixture was filtered through celite and the separated zinc washed with diethyl ether $(20 \mathrm{~mL})$ and dichloromethane $(20$ $\mathrm{mL}$ ). The organic solvents were removed in vacuo and the aqueous layer was extracted with diethyl ether $(3 \times 20 \mathrm{~mL})$. The combined organic extracts were washed with brine $(20 \mathrm{~mL})$, dried $\left(\mathrm{MgSO}_{4}\right)$, filtered and concentrated in vacuo. The crude product was then purified by flash column chromatography to give the amidocyclopropane(s). 


\section{General procedure for cyclopropanation of alkenes using $\mathrm{Zn} / \mathrm{CuCl}$}

A solution of diethoxymethylamide $(2.12 \mathrm{mmol})$ in diethyl ether $(3 \mathrm{~mL})$ was added dropwise via syringe pump $\left(0.6 \mathrm{mLh}^{-1}\right)$ to a vigorously stirred refluxing mixture of zinc dust $(1.4 \mathrm{~g}$, $21.5 \mathrm{mmol})$, copper (I) chloride (140 mg, $1.4 \mathrm{mmol})$, zinc chloride (1.0 M solution in $\mathrm{Et}_{2} \mathrm{O}, 1$ $\mathrm{mL}, 1 \mathrm{mmol})$, alkene $(1.06 \mathrm{mmol})$ and chlorotrimethlysilane $(1.4 \mathrm{~mL}, 11 \mathrm{mmol})$ in diethyl ether $(5 \mathrm{~mL})$. The reaction mixture was refluxed overnight, then quenched by addition of saturated sodium bicarbonate solution $(4 \mathrm{~mL})$, and filtered, washing the resulting solids with ether $(10 \mathrm{~mL})$. The biphasic mixture was extracted with ether $(3 \times 20 \mathrm{~mL})$ and the combined organic layers washed with brine $(30 \mathrm{~mL})$, dried $\left(\mathrm{MgSO}_{4}\right)$ and concentrated. The residue was purified by column chromatography to give the amidocyclopropane.

\section{General procedure for cyclopropanation of alkenes without TMSCI.}

A solution of diethoxymethylamide $(2.12 \mathrm{mmol})$ in diethyl ether $(2 \mathrm{~mL})$ was added dropwise via syringe pump $\left(0.6 \mathrm{mLh}^{-1}\right)$ to a vigorously stirred refluxing mixture of zinc dust $(1.4 \mathrm{~g}$, $21.5 \mathrm{mmol})$, copper (I) chloride $(140 \mathrm{mg}, 1.4 \mathrm{mmol})$, zinc chloride (1.0M solution in $\mathrm{Et}_{2} \mathrm{O}$, $5.4 \mathrm{~mL}, 5.4 \mathrm{mmol})$, alkene $(1.06 \mathrm{mmol})$ in diethyl ether $(1 \mathrm{~mL})$. The reaction mixture was refluxed overnight, then quenched by addition of saturated sodium bicarbonate solution (4 $\mathrm{mL})$, and filtered, washing the resulting solids with ether $(10 \mathrm{~mL})$. The biphasic mixture was extracted with ether $(3 \times 20 \mathrm{~mL})$ and the combined organic layers washed with brine $(30 \mathrm{~mL})$, dried $\left(\mathrm{MgSO}_{4}\right)$ and concentrated. The residue was purified by column chromatography to give the amidocyclopropane.

\section{Procedure for cyclopropanation of allyl benzene using oxazolidinone 7 and triethylorthoformate}

Zinc chloride $\left(1.0 \mathrm{M}\right.$ solution in $\left.\mathrm{Et}_{2} \mathrm{O}, 2.7 \mathrm{~mL}, 2.70 \mathrm{mmol}\right)$ and chlorotrimethylsilane $(2.45$ $\mathrm{mL}, 18.9 \mathrm{mmol})$ were added to a suspension of oxazolidinone $(238 \mathrm{mg}, 2.70 \mathrm{mmol})$ in ether $(5 \mathrm{~mL})$ and the solution heated to reflux for $10 \mathrm{~min}$. Zinc dust $(1.32 \mathrm{~g}, 20.3 \mathrm{mmol})$ and copper (I) chloride $(132 \mathrm{mg}, 1.32 \mathrm{mmol})$ were added followed by a solution of allyl benzene (165 $\mathrm{mg}, 1.39 \mathrm{mmol})$ in ether $(2 \mathrm{~mL})$. The reaction mixture was then heated to reflux and triethyl orthoformate $(2.3 \mathrm{~mL}, 13.5 \mathrm{mmol})$ was added dropwise via syringe pump $\left(0.25 \mathrm{mLh}^{-1}\right)$. After heating under reflux overnight, saturated sodium bicarbonate solution $(4 \mathrm{~mL})$ was added and the resulting suspension was filtered, washing the solids with ether $(10 \mathrm{~mL})$. The biphasic mixture was extracted with ether $(3 \times 20 \mathrm{~mL})$ and the combined organic layers washed with brine $(30 \mathrm{~mL})$, dried $\left(\mathrm{MgSO}_{4}\right)$ and concentrated. The residue was purified by column chromatography to give the amidocyclopropane $\mathbf{5 6}$ as a colourless oil (100 mg, $0.46 \mathrm{mmol}$, $33 \%)$.

\section{1-(2-Benzylcyclopropyl)-2-pyrrolidinone (trans and cis) 39}

Colourless oil; IR (mixture of trans and cis, film): $v_{\max } 2992,2958,1688$ (s), 1495, 1454, 1421 (s) $\mathrm{cm}^{-1}$; ${ }^{1} \mathrm{H}$ NMR (trans, $500 \mathrm{MHz}, \mathrm{CDCl}_{3}$ ): $\delta 0.75$ (ddd, $J=7.5,6.1,5.8,1 \mathrm{H}$, CHHCHN), 0.95 (ddd, $J=9.4,5.8,4.1,1 \mathrm{H}, \mathrm{CH} H \mathrm{CHN}$ ), 1.28 (dtdd, $J=9.4,6.8,6.1,3.5,1 \mathrm{H}$, $\mathrm{CHCHN}$ ), 1.86-1.93 (m, 2H, $\left.\mathrm{CH}_{2} \mathrm{CH}_{2} \mathrm{~N}\right), 2.32$ (t, J=8.0, 2H, $\left.\mathrm{CH}_{2} \mathrm{CO}\right), 2.50-2.55(\mathrm{~m}, 2 \mathrm{H}$, $\mathrm{CHN}$ and $\mathrm{CHHAr}), 2.70(\mathrm{dd}, J=14.7,6.8,1 \mathrm{H}, \mathrm{CHHAr}), 3.16\left(\mathrm{t}, J=7.0,2 \mathrm{H}, \mathrm{CH}_{2} \mathrm{~N}\right), 7.18-7.30$ $(\mathrm{m}, 5 \mathrm{H}, \mathrm{Ar}-H) ;{ }^{13} \mathrm{C}$ NMR (trans, $\left.125 \mathrm{MHz}, \mathrm{CDCl}_{3}\right): \delta 12.1,17.8,19.0,31.6,31.6,38.0,47.2$, 125.9, 128.2, 140.5, 175.8; ${ }^{\mathrm{H}} \mathrm{NMR}\left(\mathrm{cis}, 500 \mathrm{MHz}, \mathrm{CDCl}_{3}\right.$ ): $\delta 0.71$ (ddd, $J=6.4,6.0,4.6,1 \mathrm{H}$, CHHCHN), 1.00 (ddd, $J=8.9,8.0,6.0,1 \mathrm{H}, \mathrm{CH} H \mathrm{CHN}$ ), 1.34 (tddd, $J=8.9,7.1,6.4,4.9,1 \mathrm{H}$, CHCHN), 1.94-2.01 (m, 2H, $\left.\mathrm{CH}_{2} \mathrm{CH}_{2} \mathrm{~N}\right), 2.19$ (dd, $J=14.4,8.9,1 \mathrm{H}, \mathrm{CH} \mathrm{HAr}$ ), 2.38-2.42 (m, $2 \mathrm{H}, \mathrm{CH}_{2} \mathrm{CO}$ ), 2.66 (ddd, $J=8.0,7.1,4.6,1 \mathrm{H}, \mathrm{CHN}$ ) 3.02 (dd, $J=14.4,4.9,1 \mathrm{H}, \mathrm{CH} H \mathrm{Ar}$ ), 3.29$3.39\left(\mathrm{~m}, 2 \mathrm{H}, \mathrm{CH}_{2} \mathrm{~N}\right), 7.18-7.30(\mathrm{~m}, 5 \mathrm{H}, \mathrm{Ar}-\mathrm{H}) ;{ }^{3} \mathrm{C} \mathrm{NMR}\left(\right.$ cis, $\left.125 \mathrm{MHz}, \mathrm{CDCl}_{3}\right): \delta 10.3$, 18.2, 18.5, 30.4, 31.6, 33.8, 49.1, 125.8, 128.2, 141.1, 176.9; MS (FAB) $\mathrm{m} / \mathrm{z}(\%): 216(\mathrm{M}+\mathrm{H}$, 100), 124 (, 63), 91 (29); HMRS: $\mathrm{M}^{+}$, found 215.13149. $\mathrm{C}_{14} \mathrm{H}_{17} \mathrm{NO}$ requires 215.1310.

\section{1-(2-Phenylcyclopropyl)-2-pyrrolidinone (trans and cis) 40}

Colourless oil; IR (mixture of trans and cis, film): $v_{\max } 2980$ (s), 2886 (s), 1680 (s), 1499 , $1459 \mathrm{~cm}^{-1}$; ${ }^{1} \mathrm{H}$ NMR (trans, $\left.500 \mathrm{MHz}, \mathrm{CDCl}_{3}\right): \delta 1.28(\mathrm{dt}, J=7.7,6.3,1 \mathrm{H}, \mathrm{CHHCHN}), 1.39$ (ddd, $J=9.8,6.3,4.6,1 \mathrm{H}, \mathrm{CH} H \mathrm{CHN}$ ), 1.96-2.03 (m, $2 \mathrm{H}, \mathrm{CH}_{2} \mathrm{CH}_{2} \mathrm{~N}$ ), 2.17 (ddd, $J=9.9,6.3$, 3.6, 1H, CHCHN), 2.39 (t, J=8.1, 2H, $\mathrm{CH}_{2} \mathrm{CO}$ ), 2.78 (ddd, J=7.7, 4.6, 3.6, $1 \mathrm{H}, \mathrm{CHN}$ ), 3.35$3.40\left(\mathrm{~m}, 2 \mathrm{H}, \mathrm{CH}_{2} \mathrm{~N}\right), 7.10-7.19(\mathrm{~m}, 3 \mathrm{H}, \mathrm{Ar}-\mathrm{H}), 7.22-7.28(\mathrm{~m}, 2 \mathrm{H}, \mathrm{Ar}-\mathrm{H}) ;{ }^{13} \mathrm{C} \mathrm{NMR}$ (trans, $\left.125 \mathrm{MHz}, \mathrm{CDCl}_{3}\right): \delta 14.7,18.1,22.7,31.8,34.9,47.5,126.1,126.4,128.4,140.4,175.9 ;{ }^{1} \mathrm{H}$ $\operatorname{NMR}\left(\right.$ cis, $500 \mathrm{MHz}, \mathrm{CDCl}_{3}$ ): $\delta 1.37$ (ddd, $\left.J=9.1,7.8,6.8,1 \mathrm{H}, \mathrm{CH} \mathrm{HCHN}\right), 1.45-1.53(\mathrm{~m}, 1 \mathrm{H}$, 
$\left.\mathrm{CHHCH}_{2} \mathrm{~N}\right), 1.56-1.65\left(\mathrm{~m}, 2 \mathrm{H}, \mathrm{CH} H \mathrm{CHNand} \mathrm{CH} \mathrm{HCH}_{2} \mathrm{~N}\right), 2.10-2.22(\mathrm{~m}, 3 \mathrm{H}, \mathrm{CHCHN}$ and $\mathrm{CH}_{2} \mathrm{CO}$ ), 2.64 (ddd, $\left.J=9.5,8.5,4.8,1 \mathrm{H}, \mathrm{CHHN}\right), 2.79$ (td, $\left.J=7.8,4.9,1 \mathrm{H}, \mathrm{CHN}\right), 2.85$ (ddd, $J=9.5,8.0,6.8,1 \mathrm{H}, \mathrm{CH} H \mathrm{~N}), 7.07-7.14(\mathrm{~m}, 3 \mathrm{H}, \mathrm{Ar}-\mathrm{H}), 7.17-7.21(\mathrm{~m}, 2 \mathrm{H}, \mathrm{Ar}-\mathrm{H}) ;{ }^{13} \mathrm{C} \mathrm{NMR}$ (cis, $125 \mathrm{MHz}, \mathrm{CDCl}_{3}$ ): $\delta 10.7,18.0,21.7,31.5,32.4,48.0,126.1,127.8,127.9,136.8,176.7$; MS (EI) m/z (\%): $201\left(\mathrm{M}^{+}, 7\right), 172$ (21), 144 (24), 130 (69), 115 (100), 103 (72), 91 (52), 77 (74); HMRS: $\mathrm{M}^{+}$, found 201.11549. $\mathrm{C}_{13} \mathrm{H}_{15} \mathrm{NO}$ requires 201.11482.

\section{trans-1-(2-tert-Butylcyclopropyl)-2-pyrrolidinone 41}

Colourless oil; IR (film): $v_{\max } 2955$ (s), 2868, 1694 (s), 1462, 1421 (s) cm ${ }^{-1} ;{ }^{1} \mathrm{H}$ NMR (trans, $\left.500 \mathrm{MHz}, \mathrm{CDCl}_{3}\right): \delta 0.67-0.74\left(\mathrm{~m}, 2 \mathrm{H}, \mathrm{CH}_{2} \mathrm{CHN}\right), 0.81\left(\mathrm{~s}, 9 \mathrm{H}, \mathrm{C}\left(\mathrm{CH}_{3}\right)_{3}\right), 0.86$ (ddd, $J=9.9$, 6.8, 4.1, $1 \mathrm{H}, \mathrm{CHCHN}), 1.84-1.94\left(\mathrm{~m}, 2 \mathrm{H}, \mathrm{CH}_{2} \mathrm{CH}_{2} \mathrm{~N}\right), 2.29\left(\mathrm{t}, J=8.2,2 \mathrm{H}, \mathrm{CH}_{2} \mathrm{CO}\right), 2.47$ (dt, $J=7.5,4.1,1 \mathrm{H}, \mathrm{CHN}), 3.26\left(\mathrm{t}, J=7.1,2 \mathrm{H}, \mathrm{CH}_{2} \mathrm{~N}\right) ;{ }^{3} \mathrm{C}$ NMR $\left(\right.$ trans, $\left.125 \mathrm{MHz}, \mathrm{CDCl}_{3}\right): \delta 8.7$, 17.9, 28.1, 28.1, 29.0, 29.5, 31.8, 47.5, 175.8; MS (EI) m/z (\%): $181\left(\mathrm{M}^{+}, 20\right), 166(10), 124$ (100), 96 (29), 81 (23), 69 (14), 57 (12); HMRS: $\mathrm{M}^{+}$, found 181.146380. $\mathrm{C}_{11} \mathrm{H}_{19} \mathrm{NO}$ requires 181.146655 .

\section{( \pm )-1-((2S,3S)-2,3-Dibutylcyclopropyl)pyrrolidin-2-one 42}

Colourless oil; IR (film): $v_{\max } 2954$ (s), 2924 (s), 2856, 1694 (s), $1416 \mathrm{~cm}^{-1} ;{ }^{1} \mathrm{H}$ NMR (500 $\left.\mathrm{MHz}, \mathrm{CDCl}_{3}\right): \delta 0.68-0.79(\mathrm{~m}, 3 \mathrm{H}), 0.82(\mathrm{t}, J=7.0,3 \mathrm{H}, \mathrm{Me}), 0.83\left(\mathrm{t}, J=7.0,3 \mathrm{H}, \mathrm{CH}_{3}\right), 1.14-$ $1.36(\mathrm{~m}, 10 \mathrm{H}), 1.45-1.53(\mathrm{~m}, 1 \mathrm{H}), 1.87-1.96\left(\mathrm{~m}, 2 \mathrm{H}, \mathrm{CH}_{2} \mathrm{CH}_{2} \mathrm{~N}\right), 2.25(\mathrm{dd}, J=6.4,4.2,1 \mathrm{H}$, $\mathrm{CHN}), 2.32\left(\mathrm{t}, J=8.0,2 \mathrm{H}, \mathrm{CH}_{2} \mathrm{CO}\right), 3.20-3.31\left(\mathrm{~m}, 2 \mathrm{H}, \mathrm{CH}_{2} \mathrm{~N}\right) ;{ }^{13} \mathrm{C} \mathrm{NMR}\left(125 \mathrm{MHz}, \mathrm{CDCl}_{3}\right)$ : $\delta 13.9,14.0,18.8,22.4,22.5,24.1,25.5,27.5,31.0,31.6,31.6,32.5,36.6,49.0,176.6 ; \mathrm{MS}$ (EI) $m / z(\%): 237\left(\mathrm{M}^{+}, 43\right), 194(21), 180(100), 138(15), 124$ (35), 98 (53), 86 (35), 67 (13), 55 (15); HMRS: $\mathrm{M}^{+}$, found 237.2084. $\mathrm{C}_{15} \mathrm{H}_{27} \mathrm{NO}$ requires 237.20925 .

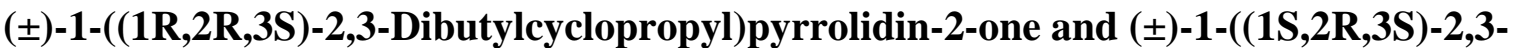 Dibutylcyclopropyl)pyrrolidin-2-one 43}

Colourless oil; IR (mixture of trans and cis, film): $v_{\max } 2956$ (s), 2929 (s), 2857, 1699 (s), $1464,1417 \mathrm{~cm}^{-1}$; ${ }^{1} \mathrm{H}$ NMR (trans, $\left.400 \mathrm{MHz}, \mathrm{CDCl}_{3}\right): \delta 0.88(\mathrm{t}, J=7.1,6 \mathrm{H}, 2 \times \mathrm{Me}), 0.98-1.02$ $(\mathrm{m}, 2 \mathrm{H}, 2 \times \mathrm{CHBu}), 1.22-1.50\left(\mathrm{~m}, 12 \mathrm{H}, 6 \times \mathrm{CH}_{2}\right), 1.98\left(\mathrm{tt}, J=8.1,7.0,2 \mathrm{H}, \mathrm{CH}_{2} \mathrm{CH}_{2} \mathrm{~N}\right), 2.01$ $(\mathrm{t}, J=3.8,1 \mathrm{H}, \mathrm{CHN}), 2.32\left(\mathrm{t}, J=8.1,2 \mathrm{H}, \mathrm{CH}_{2} \mathrm{CO}\right), 3.25\left(\mathrm{t}, J=7.0,2 \mathrm{H}, \mathrm{CH}_{2} \mathrm{~N}\right) ;{ }^{3} \mathrm{C}$ NMR (trans, $\left.100 \mathrm{MHz} \mathrm{CDCl}_{3}\right): \delta 14.1,18.0,22.6,23.5,23.5,26.8,31.8,37.8,47.4,175.6 ;{ }^{1} \mathrm{H} \mathrm{NMR}$ (cis, $\left.400 \mathrm{MHz}, \mathrm{CDCl}_{3}\right): \delta 0.88(\mathrm{t}, J=7.2,6 \mathrm{H}, 2 \times \mathrm{Me}), 0.91-0.98(\mathrm{~m}, 2 \mathrm{H}, 2 \times \mathrm{CHBu}), 1.16-1.51(\mathrm{~m}$, $\left.12 \mathrm{H}, 6 \times \mathrm{CH}_{2}\right), 1.98\left(\mathrm{tt}, J=8.2,7.7,2 \mathrm{H}, \mathrm{CH}_{2} \mathrm{CH}_{2} \mathrm{~N}\right), 2.31\left(\mathrm{t}, J=8.2,2 \mathrm{H}, \mathrm{CH}_{2} \mathrm{CO}\right), 2.38(\mathrm{t}$, $J=7.7,1 \mathrm{H}, \mathrm{CHN}), 3.25\left(\mathrm{t}, J=7.0,2 \mathrm{H}, \mathrm{CH}_{2} \mathrm{~N}\right) ;{ }^{3} \mathrm{C}$ NMR $\left(\right.$ cis, $\left.100 \mathrm{MHz}, \mathrm{CDCl}_{3}\right): \delta 14.1,18.9$, 19.8, 22.9, 23.4, 31.0, 32.4, 33.4, 48.8, 178.5; MS (EI) m/z (\%): $238(\mathrm{M}+\mathrm{H}, 100), 194$ (25), 180 (95), 138 (13), 124 (43), 98 (73), 86 (43), 67 (10), 55 (13), 41 (34); HMRS: $\mathrm{M}^{+}$, found 237.20916. $\mathrm{C}_{15} \mathrm{H}_{27} \mathrm{NO}$ requires 237.20925 .

\section{1-Bicyclo[4.1.0]hept-7-yl-2-pyrrolidinone (exo and endo) 44}

Colourless oil; IR (mixture of exo and endo, film): $v_{\max } 2927$ (s), 2853 (s), 1682 (s), 1418 (s) $\mathrm{cm}^{-1} ;{ }^{1} \mathrm{H}$ NMR $\left(\right.$ exo, $\left.500 \mathrm{MHz}, \mathrm{C}_{6} \mathrm{D}_{6}\right): \delta 0.93-1.00(\mathrm{~m}, 2 \mathrm{H}), 1.03-1.11(\mathrm{~m}, 4 \mathrm{H}), 1.21$ (tt, J=8.0, 7.0, $\left.2 \mathrm{H}, \mathrm{CH}_{2} \mathrm{CH}_{2} \mathrm{~N}\right), 1.65-1.79(\mathrm{~m}, 4 \mathrm{H}), 1.97\left(\mathrm{t}, J=8.0,2 \mathrm{H}, \mathrm{CH}_{2} \mathrm{CO}\right), 2.22(\mathrm{t}, J=3.7,1 \mathrm{H}$, $\mathrm{CHN}), 2.57$ (t, $\left.J=7.0,2 \mathrm{H}, \mathrm{CH}_{2} \mathrm{~N}\right) ;{ }^{3} \mathrm{C}$ NMR $\left(\right.$ exo, $\left.125 \mathrm{MHz}, \mathrm{C}_{6} \mathrm{D}_{6}\right): \delta 17.2,18.1,21.6,22.7$, 31.6, 36.9, 46.4, 174.4; ${ }^{1} \mathrm{H}$ NMR (endo, $\left.500 \mathrm{MHz}, \mathrm{C}_{6} \mathrm{D}_{6}\right): \delta 0.87-0.92(\mathrm{~m}, 2 \mathrm{H}, 2 \times \mathrm{CHCHN})$, $0.93-1.13(\mathrm{~m}, 4 \mathrm{H}), 1.29\left(\mathrm{tt}, J=7.9,6.9,2 \mathrm{H}, \mathrm{CH}_{2} \mathrm{CH}_{2} \mathrm{~N}\right), 1.65-1.79(\mathrm{~m}, 4 \mathrm{H}) ; 1.93(\mathrm{t}, J=7.9,2 \mathrm{H}$, $\left.\mathrm{CH}_{2} \mathrm{CO}\right), 2.04(\mathrm{t}, J=7.7,1 \mathrm{H}, \mathrm{CHN}), 2.79\left(\mathrm{t}, J=6.9,2 \mathrm{H}, \mathrm{CH}_{2} \mathrm{~N}\right) ;{ }^{13} \mathrm{C} \mathrm{NMR}$ (endo, $125 \mathrm{MHz}$, $\left.\left.\mathrm{C}_{6} \mathrm{D}_{6}\right): \delta 13.0,18.9,20.2,22.5,30.9,34.0,48.0,176.7 ; \mathrm{MS}(\mathrm{EI}) \mathrm{m} / \mathrm{z}, \%\right): 179\left(\mathrm{M}^{+}, 100\right), 150$ (32), $136(15), 124$ (20), 108 (10), 98 (32), 94 (30), 79 (19), 72 (30), 59 (57), 55 (48); HMRS: $\mathrm{M}^{+}$, found $179.13102 . \mathrm{C}_{11} \mathrm{H}_{17} \mathrm{NO}$ requires 179.1310 .

\section{1-(1-Methylbicyclo[4.1.0]hept-7-yl)-2-pyrrolidinone (exo and endo) 45}

Colourless oil; IR (mixture of exo and endo, film): $v_{\max } 2929$ (s), 2861, 1693 (s), 1492 (w), $1412 \mathrm{~cm}^{-1}$; ${ }^{1} \mathrm{H}$ NMR (mixture of exo and endo, $\left.500 \mathrm{MHz}, \mathrm{C}_{6} \mathrm{D}_{6}\right): \delta 0.73(\mathrm{td}, J=7.9,2.6,1 \mathrm{H}$, CHCHN), 0.90 (ddd, J=7.5, 4.1, 1.7, 1H, CHCHN), 0.96-1.22 (m, 14H), 1.27-1.35 (m, 4H, $\left.\mathrm{CH}_{2} \mathrm{CH}_{2} \mathrm{~N}\right), 1.47-1.66(\mathrm{~m}, 4 \mathrm{H}), 1.75-1.90(\mathrm{~m}, 5 \mathrm{H}), 1.91-1.96(\mathrm{~m}, 2 \mathrm{H}), 1.98-2.03(\mathrm{~m}, 2 \mathrm{H}), 2.09$ $(\mathrm{d}, J=4.2,1 \mathrm{H}, C \mathrm{HN}), 2.66(\mathrm{dt}, J=9.3,7.0,1 \mathrm{H}), 2.68(\mathrm{dt}, J=9.3,7.0,1 \mathrm{H}), 2.78(\mathrm{dt}, J=9.3,7.0$, $1 \mathrm{H}), 2.80(\mathrm{dt}, J=9.3,6.3,1 \mathrm{H}) ;{ }^{3} \mathrm{C}$ NMR (mixture of exo and endo, $\left.125 \mathrm{MHz}, \mathrm{C}_{6} \mathrm{D}_{6}\right): \delta 18.2$, $18.6,19.0,20.4,21.6,21.7,21.7,21.9,22.1,22.3,22.4,23.2,23.5,27.8,28.0,30.5,31.0$, 31.4, 41.2, 41.4, 47.9, 48.1, 175.2, 176.6; MS (EI) $m / z$ (\%): $193\left(\mathrm{M}^{+}, 67\right), 178$ (45), 164 (20), 
150 (15), 124 (10), 108 (83), 98 (53), 93 (55), 86 (100), 79 (18), 55 (14). HMRS: M $^{+}$, found 193.14610. $\mathrm{C}_{12} \mathrm{H}_{19} \mathrm{NO}$ requires 193.14655 .

\section{2-Ethylhexyl-[2-(2-oxopyrrolidin-1-yl)-cyclopropyl] carboxylate (trans and cis) 46}

Colourless oil; IR (diastereomeric mixture of trans and cis, film): $v_{\max } 2961$ (s), 2934 (s), 2875, 2862, 1741 (s), 1698 (s), 1460 (s), 1420 (s) cm ${ }^{-1} ;{ }^{1} \mathrm{H}$ NMR (diastereomeric mixture of trans cyclopropanes, $\left.500 \mathrm{MHz}, \mathrm{CDCl}_{3}\right): \delta 0.74\left(\mathrm{t}, J=7.2,12 \mathrm{H}, 4 \times \mathrm{CH}_{3}\right), 1.05-1.21(\mathrm{~m}, 10 \mathrm{H})$, $1.22(\mathrm{td}, J=7.6,5.5,2 \mathrm{H}), 1.26-1.51(\mathrm{~m}, 8 \mathrm{H}), 1.80-1.93\left(\mathrm{~m}, 4 \mathrm{H}, 2 \times \mathrm{CH}_{2} \mathrm{CH}_{2} \mathrm{~N}\right), 2.10$ (tt, $J=8.8,5.5,2 \mathrm{H}, 2 \times \mathrm{OCOCH}), 2.22\left(\mathrm{t}, J=8.3,4 \mathrm{H}, 2 \times \mathrm{CH}_{2} \mathrm{CO}\right), 2.57$ (ddd, $J=7.4,5.3,1.8,2 \mathrm{H}$, $2 \times \mathrm{CHN}), 3.26-3.38\left(\mathrm{~m}, 4 \mathrm{H}, 2 \times \mathrm{CH}_{2} \mathrm{~N}\right), 3.97(\mathrm{ddd}, J=7.4,4.4,1.8,2 \mathrm{H}, 2 \times \mathrm{CHOCO}) ;{ }^{13} \mathrm{C}$ NMR (diastereomeric mixture of trans cyclopropanes, $125 \mathrm{MHz}, \mathrm{CDCl}_{3}$ ): $\delta 11.5,13.6,17.8$, 22.3, 25.0, 29.2, 31.1, 31.3, 46.6, 47.0, 51.7, 175.8, 176.2, 176.7; ${ }^{1} \mathrm{H}$ NMR (diastereomeric mixture of cis cyclopropanes, $\left.300 \mathrm{MHz}, \mathrm{CDCl}_{3}\right): \delta 0.83\left(\mathrm{t}, J=7.3,12 \mathrm{H}, 4 \times \mathrm{CH}_{3}\right), 1.13-1.57$ $(\mathrm{m}, 20 \mathrm{H}), 1.89-2.01\left(\mathrm{~m}, 4 \mathrm{H}, 2 \times \mathrm{CH}_{2} \mathrm{CH}_{2} \mathrm{~N}\right), 2.19(\mathrm{tt}, J=8.2,5.6,2 \mathrm{H}, 2 \times \mathrm{OCOCH}), 2.34(\mathrm{t}$, $\left.J=8.1,4 \mathrm{H}, 2 \times \mathrm{CH}_{2} \mathrm{CO}\right), 2.65-2.70(\mathrm{~m}, 2 \mathrm{H}, 2 \times \mathrm{CHN}), 3.26-3.43\left(\mathrm{~m}, 4 \mathrm{H}, 2 \times \mathrm{CH}_{2} \mathrm{~N}\right), 4.14(\mathrm{td}$, $J=7.1,4.1,1 \mathrm{H}, 2 \times \mathrm{CHOCO}), 4.15(\mathrm{td}, J=7.1,4.1,1 \mathrm{H}, 2 \times \mathrm{CHOCO}) ;{ }^{3} \mathrm{C}$ NMR (diastereomeric mixture of cis cyclopropanes, $125 \mathrm{MHz}, \mathrm{CDCl}_{3}$ ): $\delta 11.2,11.6,13.8,18.3$, 22.5, 25.0, 25.3, 28.7, 29.4, 31.3, 31.5, 31.6, 47.0, 48.1, 49.7, 176.3, 176.5; MS (EI) $\mathrm{m} / \mathrm{z}(\%)$ : $268(\mathrm{M}+\mathrm{H}, 49), 140$ (99), 124 (32), 112 (87), 99 (28), 84 (11), 69 (42), 57 (100), 41 (73); HMRS: $\mathrm{M}+\mathrm{H}$, found 268.19116. $\mathrm{C}_{15} \mathrm{H}_{26} \mathrm{NO}_{3}$ requires 268.19124.

\section{2-Ethylhexyl-[1-methyl-2-(2-oxopyrrolidin-1-yl)-cyclopropyl] carboxylate (trans and cis) 47}

Colourless oil; IR (diastereomeric mixture of trans and cis, film): $v_{\max } 2975$ (s), 2931 (s), 2865, $1700(\mathrm{~s}), 1459(\mathrm{w}), 1419(\mathrm{w}) \mathrm{cm}^{-1} ;{ }^{1} \mathrm{H}$ NMR (diastereomeric mixture of trans and cis, $\left.500 \mathrm{MHz}, \mathrm{CDCl}_{3}\right): \delta 0.85\left(\mathrm{t}, J=7.0,24 \mathrm{H}, 8 \times \mathrm{CH}_{3}\right), 1.04(\mathrm{t}, J=6.8,2 \mathrm{H}), 1.18-1.35(\mathrm{~m}, 46 \mathrm{H})$, $1.49-1.56\left(\mathrm{~m}, 4 \mathrm{H}, 4 \times \mathrm{OCH}_{2} \mathrm{CH}\right), 1.63(\mathrm{dd}, J=8.7,5.8,2 \mathrm{H}), 1.67-1.71(\mathrm{~m}, 2 \mathrm{H}), 1.89-2.03(\mathrm{~m}$, $\left.8 \mathrm{H}, 4 \times \mathrm{CH}_{2} \mathrm{CH}_{2} \mathrm{~N}\right), 2.25-2.31\left(\mathrm{~m}, 4 \mathrm{H}, 2 \times \mathrm{CH}_{2}\right), 2.37\left(\mathrm{t}, J=8.4,4 \mathrm{H}, 2 \times \mathrm{CH}_{2}\right), 2.69(\mathrm{t}, J=6.8$, $2 \mathrm{H}, 2 \times \mathrm{CHN}), 2.99(\mathrm{dd}, J=8.5,5.7,2 \mathrm{H}, 2 \times \mathrm{CHN}), 3.20-3.36(\mathrm{~m}, 6 \mathrm{H}), 3.49(\mathrm{dt}, J=8.9,7.5$, $2 \mathrm{H}), 3.85-3.98\left(\mathrm{~m}, 8 \mathrm{H}, 4 \times \mathrm{OCH}_{2} \mathrm{CH}\right) ;{ }^{13} \mathrm{C}$ NMR (diastereomeric mixture of trans and cis, $\left.125 \mathrm{MHz} \mathrm{CDCl}_{3}\right): \delta 10.9,11.0,13.3,18.3,18.4,19.2,20.5,20.8,22.9,23.7,23.8,24.3,26.2$, $29.3,30.3,30.4,38.6,38.7,40.4,48.0,48.9,67.1,67.2,67.3,172.2,174.0,176.4,176.5 ; \mathrm{MS}$ (EI) $\mathrm{m} / \mathrm{z}(\%): 296(\mathrm{M}+\mathrm{H}, 100), 183$ (60), 165 (65), 138 (85), 98 (64), 71 (14), 57 (27), 41 (49); HMRS: $\mathrm{M}+\mathrm{H}$, found 296.22254. $\mathrm{C}_{17} \mathrm{H}_{30} \mathrm{NO}_{3}$ requires 296.22234.

\section{[2-(2-Oxopyrrolidin-1-yl)-cyclopropyl] benzoate (trans and cis) 48}

Colourless oil; IR (mixture of trans and cis, film): $v_{\max }$ 2961, 1726 (s), 1689 (s), 1615 (w), $1452,1420(\mathrm{~s}) \mathrm{cm}^{-1} ;{ }^{1} \mathrm{H}$ NMR (trans, $\left.500 \mathrm{MHz}, \mathrm{CDCl}_{3}\right): \delta 1.40-1.43(\mathrm{~m}, 2 \mathrm{H}), 1.89-1.98(\mathrm{~m}$, $\left.2 \mathrm{H}, \mathrm{CH}_{2} \mathrm{CH}_{2} \mathrm{~N}\right), 2.36\left(\mathrm{t}, J=8.4,2 \mathrm{H}, \mathrm{CH}_{2} \mathrm{CO}\right), 2.80(\mathrm{ddd}, J=7.8,7.0,5.4,1 \mathrm{H}, \mathrm{CHN}), 3.33-3.43$ $\left(\mathrm{m}, 2 \mathrm{H}, \mathrm{CH}_{2} \mathrm{~N}\right), 4.44(\mathrm{q}, J=5.4,1 \mathrm{H}, \mathrm{CHOCO}), 7.38-7.42(\mathrm{~m}, 2 \mathrm{H}, \mathrm{Ar}-\mathrm{H}), 7.51-7.56(\mathrm{~m}, 1 \mathrm{H}$, $\mathrm{Ar}-\mathrm{H})$, 7.90-7.94 (m, 2H, Ar-H); ${ }^{3} \mathrm{C}$ NMR (trans, $\left.125 \mathrm{MHz}, \mathrm{CDCl}_{3}\right): \delta 11.2,18.5,29.0,31.5$, 48.2, 50.6, 128.4, 129.3, 129.3, 133.2, 166.8, 176.7; ${ }^{1} \mathrm{H}$ NMR (cis, $\left.500 \mathrm{MHz}, \mathrm{CDCl}_{3}\right): \delta$ 1.44$1.48\left(\mathrm{~m}, 2 \mathrm{H}, \mathrm{CH}_{2} \mathrm{CHN}\right), 1.95-2.07\left(\mathrm{~m}, 2 \mathrm{H}, \mathrm{CH}_{2} \mathrm{CH}_{2} \mathrm{~N}\right), 2.37\left(\mathrm{t}, \mathrm{J}=8.4,2 \mathrm{H}, \mathrm{CH}_{2} \mathrm{CO}\right), 2.85$ (ddd, $J=9.0,6.6,1.8,1 \mathrm{H}, \mathrm{CHN}$ ), 3.45-3.53 (m, $2 \mathrm{H}, \mathrm{CH}_{2} \mathrm{~N}$ ), 4.33 (ddd, J=6.6, 5.4, 1.8, $1 \mathrm{H}$, CHO), 7.38-7.42 (m, 2H, Ar-H), 7.51-7.56 (m, 1H, Ar-H), 7.90-7.94 (m, 2H, Ar-H); ${ }^{13} \mathrm{C}$ NMR (cis, $125 \mathrm{MHz}, \mathrm{CDCl}_{3}$ ): $\delta 14.0,18.5,31.4,31.6,47.4,52.6,128.4,129.3,129.6,133.3$, 167.1, 176.2; MS (EI) m/z (\%): 245 (M+1 74$), 216$ (24), 162 (61), 154 (22), 141 (86), 124 (100), 114 (100), 106 (89), 94 (87), 84 (90), 77 (86); HMRS: $\mathrm{M}^{+}$, found 245.10578. $\mathrm{C}_{14} \mathrm{H}_{15} \mathrm{NO}_{3}$ requires 245.10519 .

\section{1-(2-Trimethylsilanylcyclopropyl)-2-pyrrolidinone 49}

Colourless oil; IR (film): $v_{\max } 2954$ (s), 2894, 1694 (s), 1419 (s) cm ${ }^{-1} ;{ }^{1} \mathrm{H}$ NMR (trans, 500 $\left.\mathrm{MHz}, \mathrm{CDCl}_{3}\right): \delta-0.12$ (ddd, $\left.J=11.3,8.2,5.3,1 \mathrm{H}, \mathrm{CHSi}\right),-0.06\left(\mathrm{~s}, 9 \mathrm{H}, \mathrm{Si}\left(\mathrm{CH}_{3}\right)_{3}\right), 0.63$ (ddd, $J=8.2,6.5,4.8,1 \mathrm{H}, \mathrm{CHHCHN}), 0.89$ (ddd, $J=11.3,4.8,3.6,1 \mathrm{H}, \mathrm{CH} H \mathrm{CHN}), 1.84-1.94(\mathrm{~m}$, $\left.2 \mathrm{H}, \mathrm{CH}_{2} \mathrm{CH}_{2} \mathrm{~N}\right), 2.31\left(\mathrm{t}, J=8.0,2 \mathrm{H}, \mathrm{CH}_{2} \mathrm{CO}\right), 2.50(\mathrm{ddd}, J=6.5,5.3,3.6,1 \mathrm{H}, \mathrm{CHN}), 3.18-3.22$ $\left(\mathrm{m}, 2 \mathrm{H}, \mathrm{CH}_{2} \mathrm{~N}\right) ;{ }^{13} \mathrm{C} \mathrm{NMR}\left(\right.$ trans, $\left.125 \mathrm{MHz}, \mathrm{CDCl}_{3}\right): \delta-2.6,4.2,8.6,18.0,29.1,31.2,47.2$, 175.8; MS (EI) m/z (\%): $197\left(\mathrm{M}^{+}, 40\right), 182$ (66), 168 (36), 154 (27), 142 (66), 124 (11), 73 (100), 59 (22); HMRS: $\mathrm{M}^{+}$, found 197.12332. $\mathrm{C}_{10} \mathrm{H}_{19} \mathrm{NOSi}$ requires 197.12355.

\section{1-[2-(Benzyldimethylsilanyl)-cyclopropyl)]-2-pyrrolidinone 50}


Colourless oil; IR (film): $v_{\max } 2953$ (m), 2891 (w), 1689 (s), 1493 (m), 1419 (m) cm ${ }^{-1}$; ${ }^{1} \mathrm{H}$ NMR $\left(500 \mathrm{MHz}, \mathrm{CDCl}_{3}\right): \delta-0.10(\mathrm{~s}, 3 \mathrm{H}, \mathrm{SiMe}),-0.08$ (ddd, $J=11.3,8.2,5.3,1 \mathrm{H}, \mathrm{CHSi}$ ), -0.05 (s, 3H, SiMe), 0.64 (ddd, $J=8.2,6.7,5.0,1 \mathrm{H}, \mathrm{CH} \mathrm{HCHN}$ ), 0.92 (ddd, $J=11.3,5.0,3.7$, $1 \mathrm{H}, \mathrm{CH} H \mathrm{CHN}), 1.87-1.95\left(\mathrm{~m}, 2 \mathrm{H}, \mathrm{CH}_{2} \mathrm{CH}_{2} \mathrm{~N}\right), 2.13\left(\mathrm{~s}, 2 \mathrm{H}, \mathrm{CH}_{2} \mathrm{Ph}\right), 2.34(\mathrm{t}, \mathrm{J}=8.0,2 \mathrm{H}$, $\mathrm{CH}_{2} \mathrm{CO}$ ), 2.61 (ddd, $\left.J=6.7,5.3,3.7,1 \mathrm{H}, \mathrm{CHN}\right), 3.16\left(\mathrm{t}, J=7.0,2 \mathrm{H}, \mathrm{CH}_{2} \mathrm{~N}\right), 6.99-7.06(\mathrm{~m}, 3 \mathrm{H}$, $\mathrm{Ar}-H)$, 7.15-7.20 (m, 2H, Ar-H); ${ }^{3} \mathrm{C}$ NMR (125 MHz, $\left.\mathrm{CDCl}_{3}\right): \delta-4.8,-4.6,2.7,8.4,18.0$, 25.2, 29.1, 31.9, 47.0, 124.0, 128.1, 128.2, 139.8, 175.9; MS (EI) $m / z(\%): 273\left(\mathrm{M}^{+}, 62\right), 258$ (24), 218 (24), 182 (92), 149 (100), 121 (86), 97 (31), 91 (23); HMRS: $\mathrm{M}^{+}$, found 273.15083. $\mathrm{C}_{16} \mathrm{H}_{23} \mathrm{NOSi}$ requires 273.15488 .

\section{trans-2-(2-Oxopyrrolidin-1-yl)cyclopropyl)methylbenzoate 51}

Colourless oil; IR(film): $v_{\max }$ 2924, 1786 (s), 1717 (s), 1682 (s) $\mathrm{cm}^{-1} ;{ }^{1} \mathrm{H}$ NMR (trans, 300 $\left.\mathrm{MHz}, \mathrm{CDCl}_{3}\right): 0.94(\mathrm{~m}, 1 \mathrm{H}, \mathrm{CHHCHN}), 1.10(\mathrm{~m}, 1 \mathrm{H}, \mathrm{CHHCHN}), 1.56\left(\mathrm{~m}, 1 \mathrm{H}, \mathrm{CHCH}_{2} \mathrm{O}\right)$, $1.97\left(\mathrm{~m}, 2 \mathrm{H}, \mathrm{CH}_{2} \mathrm{CH}_{2} \mathrm{~N}\right), 2.37,\left(\mathrm{t}, J=8.0,2 \mathrm{H}, \mathrm{CH}_{2} \mathrm{CON}\right), 2.67(\mathrm{dt}, J=7.6,3.7,1 \mathrm{H}, \mathrm{CHN}), 3.31$ $\left(\mathrm{t}, J=7.0,2 \mathrm{H}, \mathrm{CH}_{2} \mathrm{~N}\right), 4.17\left(\mathrm{dd}, J=11.6,7.6,1 \mathrm{H}, \mathrm{CHHO}_{2} \mathrm{Ph}\right), 4.32(\mathrm{dd}, J=11.6,6.6,1 \mathrm{H}$, $\left.\mathrm{CH} \mathrm{HO}_{2} \mathrm{CPh}\right), 7.44(\mathrm{dd}, J=7.5,7.9,2 \mathrm{H}$, meta-Ar), $7.56(\mathrm{t}, J=7.5,1 \mathrm{H}$, para-Ar), $8.06(\mathrm{~d}, J=7.9$, $2 \mathrm{H}$, ortho-Ar); ${ }^{13} \mathrm{C}\left(\right.$ trans, $\left.75 \mathrm{MHz}, \mathrm{CDCl}_{3}\right): 10.6,17.3,18.0,30.6,31.7,47.5,66.6,128.4$, 129.6, 130.2, 133.0, 166.6, 176.0; MS (CI+) $\mathrm{m} / z(\%): 260(\mathrm{M}+\mathrm{H}, 70), 138$ (100); HRMS: $\mathrm{M}+\mathrm{H}$, found: $260.12885 . \mathrm{C}_{15} \mathrm{H}_{18} \mathrm{NO}_{3}$ requires 260.12866 .

\section{endo-1-(1,1a,6,6a-Tetrahydro-cyclopropainden-1-yl)-pyrrolidin-2-one 52}

White needles; Mp 53-54 ${ }^{\circ} \mathrm{C}\left(\mathrm{CH}_{2} \mathrm{Cl}_{2}\right.$-Petrol); IR (film): $v_{\max } 3036$ (s), 2965 (s), 2912 (s), 2872 (s), 1693 (s) $\mathrm{cm}^{-1}$; ${ }^{\mathrm{H}} \mathrm{NMR}\left(\right.$ exo, $\left.300 \mathrm{MHz}, \mathrm{CDCl}_{3}\right): 1.12-1.26\left(\mathrm{~m}, 1 \mathrm{H}, \mathrm{CHHCH}_{2} \mathrm{~N}\right)$, 1.62 (ddddd, $J=11.6,8.8,7.9,4.7,3.5,1 \mathrm{H}, \mathrm{CHHCH}_{2} \mathrm{~N}$ ), 2.05-2.15 (m, 2H, $\mathrm{CH}_{2} \mathrm{CO}$ ), 2.25$2.33\left(\mathrm{~m}, 1 \mathrm{H}, \mathrm{CH}_{2} \mathrm{CH}\right), 2.45(\mathrm{ddd}, J=9.4,8.6,3.5,1 \mathrm{H}, \mathrm{CHHN}), 2.68(\mathrm{dd}, J 7.1,6.5,1 \mathrm{H}$, $\mathrm{ArCH}$ ), 2.77 (br t, $J=7.1,1 \mathrm{H}, \mathrm{CHN}), 2.99$ (dt, $J=9.4,7.9,1 \mathrm{H}, \mathrm{CH} H \mathrm{~N}), 3.05-3.12(\mathrm{~m}, 2 \mathrm{H}$, $\left.\mathrm{ArCH}_{2}\right), 7.03-7.10(\mathrm{~m}, 3 \mathrm{H}, \mathrm{Ar}-\mathrm{H}), 7.29-7.34(\mathrm{~m}, 1 \mathrm{H}, \mathrm{Ar}-\mathrm{H}) ;{ }^{13} \mathrm{C}$ NMR (endo, $125 \mathrm{MHz}$, $\left.\mathrm{CDCl}_{3}\right): 18.8,23.2,28.6,31.1,32.2,34.8,47.4,123.7,124.9,125.7,126.2,139.7,144.1$, 177.5. MS (CI+) m/z (\%): 214 (M+H, 100), 129 (60), 98 (42), 86 (40); HRMS: M+H, found: 214.12298. $\mathrm{C}_{14} \mathrm{H}_{16} \mathrm{NO}$ requires 214.12318 .

\section{(endo and exo) 1-(1a,2,3,7b-Tetrahydro-1H-cyclopropa[a]naphthalen-1-yl)-pyrrolidin-2-} one 53

Colourless oil; IR (film): $v_{\max }$ 2926, 2856, 1693 (s) $\mathrm{cm}^{-1} ;{ }^{1} \mathrm{H}$ NMR (trans, $500 \mathrm{MHz}, \mathrm{CDCl}_{3}$ ): (mixture of isomers) $1.40-3.05\left(\mathrm{~m}, 12 \mathrm{H}, \mathrm{CH}_{2}\right.$ and $\left.\mathrm{CH}\right), 3.25-3.42(\mathrm{~m}, 1 \mathrm{H}), 6.90-7.15(\mathrm{~m}, 3 \mathrm{H}$, Ar- $H$ ), 7.20-7.35 (m, $1 \mathrm{H}, \mathrm{Ar}-\mathrm{H}) ;{ }^{13} \mathrm{C}$ NMR (mixture of isomers, $125 \mathrm{MHz}, \mathrm{CDCl}_{3}$ ): 17.9, 18.0, 18.1, 18.6, 18.9, 19.5, 21.1, 22.3, 26.0, 27.4, 31.0, 31.7, 34.0, 37.3, 47.3, 47.7, 125.5, $125.8,125.9,126.1,128.5,128.6,128.7,130.3,133.2,133.9,135.4,135.5,175.7,177.7 ; \mathrm{MS}$ (EI) $m / z(\%): 227\left(\mathrm{M}^{+}, 10\right), 142$ (100), 128 (25), 115 (20), 99 (62), 86 (45); HRMS: $\mathbf{M}^{+}$, found: 227.13122 . $\mathrm{C}_{15} \mathrm{H}_{17} \mathrm{NO}$ requires 227.13101 .

\section{Ethyl 2-cis-((2-(2-oxopyrrolidin-1-yl)cyclopropyl)methoxy)acetate 54}

Colourless oil; IR (film): $v_{\max }$ 2980, 1751 (s), 1690 (s) cm ${ }^{-1} ;{ }^{1} \mathrm{H}$ NMR (cis, $300 \mathrm{MHz}, \mathrm{CDCl}_{3}$ ); $0.92(\mathrm{td}, J=6.3,4.7,1 \mathrm{H}, \mathrm{CHHCHN}), 1.08$ (ddd, $J=9.0,7.7,6.3,1 \mathrm{H}, \mathrm{CH} H \mathrm{CHN}), 1.27(\mathrm{t}$, $J=7.1,3 \mathrm{H}, \mathrm{Me}), 1.35-1.47(\mathrm{~m}, 1 \mathrm{H}, \mathrm{C} H \mathrm{CHN}), 1.91-2.03\left(\mathrm{~m}, 2 \mathrm{H}, \mathrm{CH}_{2} \mathrm{CH}_{2} \mathrm{~N}\right), 2.33-2.41(\mathrm{~m}$, $2 \mathrm{H}, \mathrm{CH}_{2} \mathrm{CO}$ ), 2.54-2.62 (ddd, $\left.J=7.7,6.8,4.7,1 \mathrm{H}, \mathrm{CHN}\right), 3.31(\mathrm{dt}, J=14.7,7.0,1 \mathrm{H}, \mathrm{CH} \mathrm{HN}$ ), 3.37 (dd, $J=10.0,6.8,1 \mathrm{H}, \mathrm{CHHOR}), 3.44-3.57(\mathrm{~m}, 1 \mathrm{H}, \mathrm{CH} H \mathrm{~N}), 3.62$ (dd, $J=10.0,7.5,1 \mathrm{H}$, $\mathrm{CH} H \mathrm{OR}), 4.05-4.25\left(\mathrm{~m}, 4 \mathrm{H}, \mathrm{CH}_{2} \mathrm{Me}\right.$ and $\left.\mathrm{CH}_{2} \mathrm{CO}_{2} \mathrm{Et}\right) ;{ }^{13} \mathrm{C} \mathrm{NMR}$ (cis, $\left.125 \mathrm{MHz}, \mathrm{CDCl}_{3}\right): 8.6$, $13.9,16.6,18.1,29.5,31.4,48.8,60.5,67.6,70.2,170.0,176.9 ; \mathrm{MS}(\mathrm{CI}+) \mathrm{m} / \mathrm{z}(\%): 242$ $(\mathrm{M}+\mathrm{H}, 71), 214$ (39), 195 (31), 138 (100); HRMS: $\mathrm{M}+\mathrm{H}$, found: 242.13896. $\mathrm{C}_{12} \mathrm{H}_{20} \mathrm{NO}_{4}$ requires 242.13923 .

\section{1-(2,2,3,3-Tetramethylcyclopropyl)pyrrolidin-2-one 55}

Colourless oil; IR (film): $v_{\max } 2924$ (s), 2870 (s), 1682 (s) $\mathrm{cm}^{-1} ;{ }^{1} \mathrm{H}$ NMR (400 MHz, $\mathrm{CDCl}_{3}$ ): $0.99(\mathrm{~s}, 6 \mathrm{H}, 2 \times \mathrm{Me}), 1.08(\mathrm{~s}, 6 \mathrm{H}, 2 \times \mathrm{Me}), 1.72(\mathrm{~s}, 1 \mathrm{H}, \mathrm{CHN}), 1.94\left(\mathrm{~m}, 2 \mathrm{H}, \mathrm{CH}_{2}\right), 2.28(\mathrm{t}$, $\left.J=8.0,2 \mathrm{H}, \mathrm{CH}_{2} \mathrm{CO}\right), 3.29\left(\mathrm{t}, J 6.9,2 \mathrm{H}, \mathrm{CH}_{2} \mathrm{~N}\right) ;{ }^{13} \mathrm{C}$ NMR $\left(100 \mathrm{MHz}, \mathrm{CDCl}_{3}\right): 17.6,18.8,22.0$, 23.2, 31.0, 46.1, 48.6, 177.9; MS (EI) $\mathrm{m} / z(\%): 182(\mathrm{M}+\mathrm{H}, 100), 166$ (15), 154 (15); HRMS: $\mathrm{M}+\mathrm{H}$, found: $182.15411 . \mathrm{C}_{11} \mathrm{H}_{21} \mathrm{NO}$ requires 182.15448 . 
Colourless oil; IR (mixture of trans and cis, film): $v_{\max } 3003$ (w), 2915 (w), 1742 (s), 1604 (w), $1482(\mathrm{w}), 1419(\mathrm{w}) \mathrm{cm}^{-1} ;{ }^{1} \mathrm{H}$ NMR (trans, $\left.500 \mathrm{MHz}, \mathrm{CDCl}_{3}\right): \delta 0.82(\mathrm{dt}, J=7.1,6.1,1 \mathrm{H}$, CHHCHN), 1.03 (ddd, J=9.5, 6.1, 3.5, 1H, CHHCHN), 1.36 (dtdd, J=9.5, 6.9, 6.1, 3.5, $1 \mathrm{H}$, CHCHN), $2.40(\mathrm{dt}, J=7.1,3.5,1 \mathrm{H}, \mathrm{CHN}), 2.59$ (dd, $J=14.5,6.9,1 \mathrm{H}, \mathrm{C} H \mathrm{HPh}), 2.72$ (dd, $J=14.5,6.9,1 \mathrm{H}, \mathrm{CH} H \mathrm{Ph}), 3.38-3.42\left(\mathrm{~m}, 2 \mathrm{H}, \mathrm{CH}_{2} \mathrm{~N}\right), 4.19-4.25\left(\mathrm{~m}, 2 \mathrm{H}, \mathrm{CH}_{2} \mathrm{O}\right), 7.20-7.32$ $(\mathrm{m}, 5 \mathrm{H}, \mathrm{Ar}-\mathrm{H}) ;{ }^{13} \mathrm{C} \mathrm{NMR}\left(\right.$ trans, $\left.125 \mathrm{MHz}, \mathrm{CDCl}_{3}\right): \delta 13.0,20.3,32.1,37.8,45.7,61.8$, 126.2, 128.2, 128.3, 140.5, 158.4: ${ }^{1} \mathrm{H}$ NMR (cis, $\left.500 \mathrm{MHz}, \mathrm{CDCl}_{3}\right): \delta 0.78(\mathrm{td}, J=6.3,4.2,1 \mathrm{H}$, CHHCHN), 1.05-1.15 (m, 2H, CHHCHN and CHCHN), 2.44 (dd, $J=15.4,9.2,1 \mathrm{H}, \mathrm{CHHPh}$ ), 2.65 (ddd, $J=7.7,6.9,4.2,1 \mathrm{H}, \mathrm{CHN}), 3.11$ (dd, $J=15.4,5.1,1 \mathrm{H}, \mathrm{CH} H \mathrm{Ph}), 3.51-3.57$ (m, 2H, $\left.\mathrm{CH}_{2} \mathrm{~N}\right), 4.28-4.33\left(\mathrm{~m}, 2 \mathrm{H}, \mathrm{C} \mathrm{H}_{2} \mathrm{O}\right), 7.20-7.32(\mathrm{~m}, 5 \mathrm{H}$, Ar- $\mathrm{H}) ;{ }^{13} \mathrm{C} \mathrm{NMR}($ cis, $100 \mathrm{MHz}$, $\left.\mathrm{CDCl}_{3}\right): \delta 11.1,18.7,31.0,33.7,46.9,62.0,126.0,128.2,141.0,159.7 ; \mathrm{MS}(\mathrm{CI}+) \mathrm{m} / \mathrm{z}(\%)$ : $218(\mathrm{M}+\mathrm{H}, 17), 126$ (100), 115 (11), 104 (15), 91 (22), 82 (12), 77 (7), 65 (8), 54 (9) ; HMRS: $\mathrm{M}+\mathrm{H}$, found 218.11777. $\mathrm{C}_{13} \mathrm{H}_{16} \mathrm{NO}_{2}$ requires 218.11809 .

\section{3-(2-tert-Butylcyclopropyl)-2-oxazolidinone 57}

Pale yellow oil; IR (film): $v_{\max } 2958,2869$ (s), 1755 (s), 1482 (w), $1423 \mathrm{~cm}^{-1}$; ${ }^{1} \mathrm{H}$ NMR (trans, $500 \mathrm{MHz}, \mathrm{CDCl}_{3}$ ): $\delta 0.75$ (td, $\left.J=7.3,5.9,1 \mathrm{H}, \mathrm{CHHCHN}\right), 0.80$ (ddd, $J=9.8,5.9,3.7,1 \mathrm{H}$, $\mathrm{CH} H \mathrm{CHN}), 0.85(\mathrm{~s}, 9 \mathrm{H}, 3 \times \mathrm{Me}), 0.95$ (ddd, $J=9.8,7.3,3.7,1 \mathrm{H}, \mathrm{CHCHN}), 2.35$ (dt, $J=7.3$, 3.7, $1 \mathrm{H}, \mathrm{CHN}), 3.45-3.50\left(\mathrm{~m}, 2 \mathrm{H}, \mathrm{CH}_{2} \mathrm{~N}\right), 4.22\left(\mathrm{~m}, 2 \mathrm{H}, \mathrm{CH}_{2} \mathrm{O}\right) ;{ }^{3} \mathrm{C}$ NMR $\left(125 \mathrm{MHz}, \mathrm{CDCl}_{3}\right)$ : $\delta$ 9.4, 28.2, 28.7, 29.0, 30.6, 45.8, 61.6, 158.4; MS (EI) m/z (\%): $183\left(\mathrm{M}^{+}, 7\right), 168(6), 127$ (100), 114 (31), 82 (18), 70 (31), 55 (43), 49 (12), 42 (60); HMRS: M+H, found 184.13305. $\mathrm{C}_{10} \mathrm{H}_{18} \mathrm{NO}_{2}$ requires 184.13321 .

( \pm )-Ethyl 2-((1R,2S,5S,6R)-6-(2-oxooxazolidin-3-yl)bicyclo[3.1.0]hexan-2-yl)acetate 58a and $( \pm)$-Ethyl 2-((1R,2R,5S,6R)-6-(2-oxooxazolidin-3-yl)bicyclo[3.1.0]hexan-2-yl)acetate $\mathbf{5 8 b}$

Pale yellow oil; IR (mixture of 58a and 58b , film): $v_{\max } 2954,1732(\mathrm{~s}), 1417(\mathrm{~s}) \mathrm{cm}^{-1} ;{ }^{1} \mathrm{H}$ NMR (major isomer 58a, $\left.500 \mathrm{MHz}, \mathrm{CDCl}_{3}\right): \delta 1.20\left(\mathrm{t}, J=7.2,3 \mathrm{H}, \mathrm{CH}_{3}\right), 1.28-1.33(\mathrm{~m}, 1 \mathrm{H}$, $\left.\mathrm{CHHCHCH}_{2}\right), 1.35-1.45(\mathrm{~m}, 1 \mathrm{H}, \mathrm{CH} H \mathrm{CHCH} 2), 1.45-1.49(\mathrm{~m}, 1 \mathrm{H}, \mathrm{CHCHN}), 1.69-1.72(\mathrm{~m}$, $1 \mathrm{H}, \mathrm{CHCHN}), 1.78-1.84(\mathrm{~m}, 1 \mathrm{H}, \mathrm{CH} \mathrm{HCHCHN}), 1.84-1.91(\mathrm{~m}, 1 \mathrm{H}, \mathrm{CH} H \mathrm{CHCHN}), 2.20$ (dd, $J=15.1,8.2,1 \mathrm{H}, \mathrm{CHHCOO}), 2.28(\mathrm{t}, J=1.9,1 \mathrm{H}, \mathrm{CHN}), 2.32$ (dd, $J=15.1,7.1,1 \mathrm{H}, \mathrm{CH} H \mathrm{COO}$ ), 2.62 (br q, $\left.J=7.5,1 \mathrm{H}, \mathrm{CHCH}_{2} \mathrm{CO}\right), 3.47-3.51\left(\mathrm{~m}, 2 \mathrm{H}, \mathrm{CH}_{2} \mathrm{~N}\right), 4.14\left(\mathrm{q}, J=7.2,2 \mathrm{H}, \mathrm{CH}_{2} \mathrm{CH}_{3}\right)$, 4.22-4.27 (m, 2H, CH $2 \mathrm{OCON}$ ): ${ }^{13} \mathrm{C}$ NMR (major isomer 58a, $\left.125 \mathrm{MHz}, \mathrm{CDCl}_{3}\right): \delta 14.2,24.7$, 24.8, 27.0, 29.7, 33.4, 36.5, 39.8, 45.7, 60.3, 61.8, 158.3, $172.5 ;{ }^{1} \mathrm{H}$ NMR (minor isomer 58b,

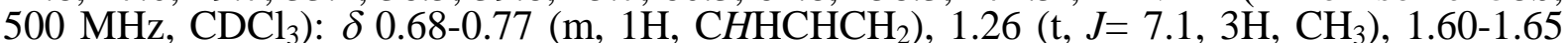
$(\mathrm{m}, 1 \mathrm{H}, \mathrm{CHCHN}), 1.69-1.73(\mathrm{~m}, 1 \mathrm{H}, \mathrm{CHCHN}), 1.73-1.79\left(\mathrm{~m}, 1 \mathrm{H}, \mathrm{CH} H \mathrm{CHCH}{ }_{2}\right), 1.79-1.88$ $(\mathrm{m}, 1 \mathrm{H}, \mathrm{CH} \mathrm{HCHCHN}), 1.95$ (dd, $J=12.9,8.1,1 \mathrm{H}, \mathrm{CH} H \mathrm{CHCHN}$ ), 2.28 (br t $, J=1.9,1 \mathrm{H}$, $\mathrm{CHN}), 2.40(\mathrm{dd}, J=15.2,7.1,1 \mathrm{H}, \mathrm{CH} \mathrm{HCOO}), 2.46(\mathrm{dd}, J=15.2,7.6,1 \mathrm{H}, \mathrm{CH} H \mathrm{COO}), 2.56-$ $2.65\left(\mathrm{~m}, 1 \mathrm{H}, \mathrm{CHCH}_{2} \mathrm{CO}\right), 3.43-3.51\left(\mathrm{~m}, 2 \mathrm{H}, \mathrm{CH}_{2} \mathrm{~N}\right), 4.14\left(\mathrm{q}, J=7.1,2 \mathrm{H}, \mathrm{CH}_{2} \mathrm{CH}_{3}\right), 4.19-4.28$ $\left(\mathrm{m}, 2 \mathrm{H}, \mathrm{CH}_{2} \mathrm{OCON}\right.$ ); ${ }^{13} \mathrm{C} \mathrm{NMR}$ (minor isomer 58b, $125 \mathrm{MHz}, \mathrm{CDCl}_{3}$ ): $\delta 14.3,25.5,27.0$, 27.6, 28.9, 30.8, 36.6, 37.8, 45.7, 60.3, 61.8, 158.4, 173.0 ; MS (CI+) $\mathrm{m} / \mathrm{z}(\%): 254(\mathrm{M}+\mathrm{H}$, 100), 208 (95), 166 (60), 88 (14); HMRS: $\mathrm{M}+\mathrm{H}$, found $254.13872, \mathrm{C}_{13} \mathrm{H}_{20} \mathrm{O}_{4} \mathrm{~N}$ requires 254.13923 .

\section{Methyl 3-(1-chloro-2-(2-oxooxazolidin-3-yl)cyclopropyl)-2,2-dimethylpropanoate 59 22}

White solid; m.p: $46-49{ }^{\circ} \mathrm{C}$ (petrol); IR (mixture of trans and cis, film): $v_{\max } 1634$ (s), 1427 (w) $\mathrm{cm}^{-1} ;{ }^{1} \mathrm{H}$ NMR (trans, $\left.500 \mathrm{MHz}, \mathrm{CDCl}_{3}\right): \delta 1.28\left(\mathrm{~s}, 3 \mathrm{H}, \mathrm{CH}_{3} \mathrm{CCH}_{3}\right), 1.29(\mathrm{~s}, 3 \mathrm{H}$, $\left.\mathrm{CH}_{3} \mathrm{CCH}_{3}\right), 1.36$ (dd, $\left.J=9.0,7.9,1 \mathrm{H}, \mathrm{CH} \mathrm{HCHN}\right) 1.59$ (ddd, $\left.J=7.9,5.5,1.0,1 \mathrm{H}, \mathrm{CH} H \mathrm{CHN}\right)$, $2.01(\mathrm{~d}, J=15.2,1 \mathrm{H}, \mathrm{CHHCCHN}), 2.11(\mathrm{dd}, J=15.2,1.0,1 \mathrm{H}, \mathrm{CH} H \mathrm{CCHN}), 2.51$ (dd, $J=9.0$, 5.5, $1 \mathrm{H}, \mathrm{CHN}), 3.56-3.64(\mathrm{~m}, 1 \mathrm{H}, \mathrm{CH} \mathrm{HN}), 3.68\left(\mathrm{~s}, 3 \mathrm{H}, \mathrm{CH}_{3} \mathrm{O}\right), 3.74-3.80(\mathrm{~m}, 1 \mathrm{H}, \mathrm{CH} H \mathrm{~N})$, 4.25-4.37 (m, 2H, $\left.\mathrm{CH}_{2} \mathrm{OCO}\right) ;{ }^{13} \mathrm{C} \mathrm{NMR}\left(\right.$ cis, $\left.125 \mathrm{MHz}, \mathrm{CDCl}_{3}\right): \delta 22.0,25.8,25.8,36.7,42.2$, 44.5, 45.6, 48.5, 51.9, 61.9, 158.5, 177.1; MS (CI+) m/z (\%): $276(\mathrm{M}+\mathrm{H}, 14), 244$ (28), 216 (100), 180 (16); HMRS: $\mathrm{M}+\mathrm{H}$, found 276.09988, $\mathrm{C}_{12} \mathrm{H}_{19} \mathrm{O}_{4} \mathrm{NCl}$ requires 276.10025.

\section{trans-8-endo-(2-Oxooxazolidin-3-yl)-1,4,4-trimethyl-tricyclo[5.1.0.0]octane 60}

White solid; m.p: $60-64{ }^{\circ} \mathrm{C}$ (petrol); $[\alpha]^{20}=-27.1$ (c 1.0, $\mathrm{CHCl}_{3}$ ); IR (mixture , film): $v_{\max }$ $2864(\mathrm{w}), 1755,1634,1404 \mathrm{~cm}^{-1}$; ${ }^{\mathrm{H}} \mathrm{NMR}\left(\right.$ endo, $\left.500 \mathrm{MHz}, \mathrm{CDCl}_{3}\right): \delta 0.37(\mathrm{td}, J=9.4,3.2$, $1 \mathrm{H}, \mathrm{CHCMe} 2), 0.43(\mathrm{td}, J=9.4,3.2,1 \mathrm{H}, \mathrm{CHCMe}), 0.77\left(\mathrm{~s}, 3 \mathrm{H}, \mathrm{CH}_{3} \mathrm{CCHN}\right), 0.82(\mathrm{~m}, 1 \mathrm{H}$, $\mathrm{CHCHN}), 0.98\left(\mathrm{~s}, 3 \mathrm{H}, \mathrm{CH}_{3} \mathrm{CCH}_{3}\right), 1.02\left(\mathrm{~s}, 3 \mathrm{H}, \mathrm{CH}_{3} \mathrm{CCH}_{3}\right), 1.30(\mathrm{dd}, J=15.7,3.2,1 \mathrm{H}$, $\left.\mathrm{CHHCCH}_{3}\right), 1.63$ (ddd, $\left.J=15.7,5.8,3.2,1 \mathrm{H}, \mathrm{CHHCHCHN}\right), 2.08$ (d, $\left.J=7.7,1 \mathrm{H}, \mathrm{CHN}\right), 2.27$ (dd, $\left.J=15.7,9.4,1 \mathrm{H}, \mathrm{CH} H \mathrm{CCH}_{3}\right), 2.28$ (dd, $\left.J=15.7,9.4,1 \mathrm{H}, \mathrm{CH} H \mathrm{CHCHN}\right), 3.63$ (t, $J=7.8$, 
$2 \mathrm{H}, \mathrm{CH}_{2} \mathrm{~N}$ ), 4.24-4.28 (m, 2H, $\left.\mathrm{CH}_{2} \mathrm{O}\right) ;{ }^{13} \mathrm{C} \mathrm{NMR}$ (cis, $\left.125 \mathrm{MHz}, \mathrm{CDCl}_{3}\right): \delta 13.9,15.1,16.1$, $16.9,17.1,17.7,20.3,21.3,26.5,27.8,38.1,46.5,62.4,159.8 ; \mathrm{MS}(\mathrm{CI}+) \mathrm{m} / \mathrm{z}(\%): 236$ $(\mathrm{M}+\mathrm{H}, 43), 149(100), 100(22), 88$ (48); HMRS: $\mathrm{M}+\mathrm{H}$, found $236.16482, \mathrm{C}_{14} \mathrm{H}_{22} \mathrm{O}_{2} \mathrm{~N}$ requires 236.16505. Anal Calcd for $\mathrm{C}_{14} \mathrm{H}_{21} \mathrm{O}_{2} \mathrm{~N} ; \mathrm{C}, 71.46 ; \mathrm{H}, 8.99 ; \mathrm{N}, 5.95$. Found: C, 71.54; H, 9.12; N, 5.90 .

\section{(E)-Ethyl 5-(2,2-dimethyl-3-(2-oxooxazolidin-3-yl)cyclopropyl)-3-methylpent-2-enoate 61}

Pale yellow liquid; IR (mixture of trans and cis, film): $v_{\max } 2955,1759,1709,1643(\mathrm{~s}) \mathrm{cm}^{-1}$; ${ }^{1} \mathrm{H}$ NMR (cis, $\left.500 \mathrm{MHz}, \mathrm{CDCl}_{3}\right): \delta 0.74$ (ddd, $\left.J=8.9,7.6,5.4,1 \mathrm{H}, \mathrm{CHCHN}\right), 1.07(\mathrm{~s}, 3 \mathrm{H}$, $\left.\mathrm{CH}_{3} \mathrm{CCH}_{3}\right), 1.09\left(\mathrm{~s}, 3 \mathrm{H}, \mathrm{CH}_{3} \mathrm{CCH}_{3}\right) 1.25$ (br t, J=7.1, 3H, $\left.\mathrm{CH}_{3} \mathrm{CH}_{2}\right), 1.34-1.44(\mathrm{~m}, 1 \mathrm{H}$, $\left.\mathrm{CHHCH}_{2}\right), 1.76-1.84\left(\mathrm{~m}, 1 \mathrm{H}, \mathrm{CH} H C H_{2}\right), 2.10(\mathrm{~d}, \mathrm{~J}=7.6,1 \mathrm{H}, \mathrm{CHN}), 2.14\left(\mathrm{~s}, 3 \mathrm{H}, \mathrm{CH}_{3} \mathrm{C}=\mathrm{CH}\right)$, 2.16-2.22 (m, 1H, CHHC=CH), 2.23-2.31 (m, $1 \mathrm{H}, \mathrm{CH} H \mathrm{C}=\mathrm{CH}), 3.43-3.56\left(\mathrm{~m}, 2 \mathrm{H}, \mathrm{CH}_{2} \mathrm{~N}\right)$, $4.12\left(\mathrm{q}, J=7.1,2 \mathrm{H}, \mathrm{CH}_{2} \mathrm{CH}_{3}\right), 4.22-4.30\left(\mathrm{~m}, 2 \mathrm{H}, \mathrm{CH}_{2} \mathrm{OC}=\mathrm{O}\right), 5.64($ br s, $1 \mathrm{H}, \mathrm{CHCOO}) ;{ }^{13} \mathrm{C}$ NMR (cis, $\left.125 \mathrm{MHz}, \mathrm{CDCl}_{3}\right): \delta 14.3,14.9,18.9,19.5,22.8,22.9,27.0,27.8,40.9,46.9,59.5$, 62.2, 115.8, 159.5, 160.4, 166.7; MS (FAB) m/z (\%): $296(\mathrm{M}+\mathrm{H}, 28), 250(70), 219$ (100), 163 (66), 154 (72); HMRS: $\mathrm{M}+\mathrm{H}$, found 296.18541, $\mathrm{C}_{16} \mathrm{H}_{26} \mathrm{O}_{4} \mathrm{~N}$ requires 296.18617.

\section{2-(2-Benzylcyclopropyl)-2,3-dihydro-1-isoindolinone 62}

Pale yellow solid; $\mathrm{Mp}$ (trans) $62-63^{\circ} \mathrm{C}\left(i-\mathrm{Pr}_{2} \mathrm{O}\right)$; IR (mixture of trans and cis, film): $\gamma_{\text {max }} 3027$ (w), $2914(\mathrm{w}), 1685(\mathrm{~s}), 1620(\mathrm{w}), 1496(\mathrm{w}), 1469(\mathrm{~m}), 1453(\mathrm{~m}), 1408(\mathrm{~m}) \mathrm{cm}^{-1}$; ${ }^{\mathrm{H}} \mathrm{NMR}$ (trans, $500 \mathrm{MHz}, \mathrm{CDCl}_{3}$ ): $\delta 0.93(\mathrm{td}, J=7.1,6.0,1 \mathrm{H}, \mathrm{CHHCHN}), 1.15$ (ddd, $J=9.6,6.0,4.0$, CHHCHN), 1.50 (dtdd, J=9.6, 7.1, 6.4, 3.4, 1H, CHCHN), 2.67 (dd, $J=14.7,7.1,1 \mathrm{H}$, $\mathrm{CH} \mathrm{HPh}$ ), 2.80-2.89 (m, 2H, CHN and $\mathrm{CH} H \mathrm{Ph}), 4.20$ (s, 2H, $\left.\mathrm{CH}_{2} \mathrm{Ar}\right), 7.20-7.53$ (m, 8H, Ar$H$ ), $7.82(\mathrm{td}, J=7.5,1.0,1 \mathrm{H}, \mathrm{Ar}-H) ;{ }^{13} \mathrm{C} \mathrm{NMR}\left(\right.$ trans, $\left.125 \mathrm{MHz}, \mathrm{CDCl}_{3}\right): \delta 12.8,20.0,31.6$, $38.2,50.1,122.5,123.4,126.2,127.9,128.4,128.4,131.2,133.2,140.6,140.9,169.3 ;{ }^{1} \mathrm{H}$ NMR (cis, $\left.500 \mathrm{MHz}, \mathrm{CDCl}_{3}\right): \delta 0.90-0.97(\mathrm{~m}, 1 \mathrm{H}), 1.11-1.19(\mathrm{~m}, 2 \mathrm{H}), 2.24(\mathrm{dd}, J=15.0,4.9$, $1 \mathrm{H}, \mathrm{CHHPh}), 3.03(\mathrm{td}, J=7.5,4.5,1 \mathrm{H}, \mathrm{CHN}), 3.15(\mathrm{dd}, J=15.0,4.9,1 \mathrm{H}, \mathrm{CH} H \mathrm{Ph}), 4.30$ (d, $J=17.0,1 \mathrm{H}, \mathrm{CHHAr}), 4.32(\mathrm{~d}, J=17.0,1 \mathrm{H}, \mathrm{CH} H \mathrm{Ar}), 7.17-7.57(\mathrm{~m}, 8 \mathrm{H}, \mathrm{Ar}-H), 7.88(\mathrm{td}$, $J=7.4,1.0,1 \mathrm{H}, \mathrm{Ar}-H) ;{ }^{3} \mathrm{C}$ NMR (cis, $\left.125 \mathrm{MHz}, \mathrm{CDCl}_{3}\right): \delta 10.6,19.2,30.5,34.0,51.8,122.6$, 123.6, 125.9, 128.0, 128.3, 128.4, 131.3, 133.1, 141.1, 141.3, 170.3; MS (EI) $\mathrm{m} / z$ (\%): 263 $\left(\mathrm{M}^{+}, 13\right), 222(8), 172(100), 146(9), 132$ (7), $115(9), 91$ (29), 77 (8), 65 (8), 51 (7); HMRS: $\mathrm{M}^{+}$, found 263.13096. $\mathrm{C}_{18} \mathrm{H}_{17} \mathrm{NO}$ requires 263.13101 .

\section{$N$-Benzyl- $N$-(2-benzylcyclopropyl)-acetamide 63}

Colourless oil; IR (mixture of trans and cis, film): $v_{\max } 3026$ (w), 2924 (w), 1655 (s), 1603 (w), $1495(\mathrm{w}), 1452(\mathrm{w}) \mathrm{cm}^{-1} ;{ }^{1} \mathrm{H}$ NMR (trans, $500 \mathrm{MHz}, \mathrm{CDCl}_{3}$ ): $\delta 0.78$ (ddd, $J=7.1,6.2$, $5.5,1 \mathrm{H}, \mathrm{CH} \mathrm{HCHN}), 0.95$ (ddd, $J=9.5,5.5,4.0,1 \mathrm{H}, \mathrm{CH} H \mathrm{CHN}), 1.37$ (ddtd, $J=9.5,7.5,6.2$, $3.5,1 \mathrm{H}, \mathrm{CHCHN}), 2.16(\mathrm{~s}, 3 \mathrm{H}, \mathrm{Me}), 2.33-2.44(\mathrm{~m}, 1 \mathrm{H}, \mathrm{CHN}), 2.42(\mathrm{dd}, J=14.5,7.5,1 \mathrm{H}$, CHCHHPh), 2.66 (dd, $J=14.5,6.2,1 \mathrm{H}, C H C H H P h), 4.45$ (d, J=14.8, 1H, CHHPh), 4.53 (d, $J=14.8,1 \mathrm{H}, \mathrm{CH} H \mathrm{Ph}), 7.08-7.12(\mathrm{~m}, 4 \mathrm{H}, \mathrm{Ar}-H), 7.19-7.31(\mathrm{~m}, 6 \mathrm{H}, \mathrm{Ar}-H) ;{ }^{13} \mathrm{C}$ NMR (trans, $\left.125 \mathrm{MHz}, \mathrm{CDCl}_{3}\right): \delta 15.9,22.7,23.3,36.8,37.7,49.6,126.3,126.9,128.4,128.5,138.1$, 139.7, 173.3; MS (EI) $\mathrm{m} / z(\%): 279\left(\mathrm{M}^{+}, 65\right), 264$ (37), 236 (100), 188 (100), 148 (74), 131 (66), 106 (52), 91 (82), 77 (24); HMRS: $\mathrm{M}^{+}$, found 279.16205. $\mathrm{C}_{19} \mathrm{H}_{21} \mathrm{NO}$ requires 279.16231.

\section{trans-3-(2-Benzylcyclopropyl)benzoxazolin-2-one 64}

Pale pink solid; $\mathrm{Mp} 55-57{ }^{\circ} \mathrm{C}\left(\mathrm{CH}_{2} \mathrm{Cl}_{2}\right.$-Petrol); IR (film): $v_{\max } 3063,3026,2924,1771$ (s), 1612 (w) cm ${ }^{-1} ;{ }^{1} \mathrm{H}$ NMR (trans, $400 \mathrm{MHz}, \mathrm{CDCl}_{3}$ ): 1.08-1.18 (m, 1H, CHHCHN), 1.30-1.42 $(\mathrm{m}, 1 \mathrm{H}, \mathrm{CH} H \mathrm{CHN}), 1.55-1.70(\mathrm{~m}, 1 \mathrm{H}, \mathrm{CHCHN}), 2.73(\mathrm{dt}, J=6.7,3.4,1 \mathrm{H}, \mathrm{CHN}), 2.76(\mathrm{dd}$, $J=14.5,7.5,1 \mathrm{H}, \mathrm{CH} H \mathrm{Hh}) 2.87(\mathrm{dd}, J=14.5,6.7,1 \mathrm{H}, \mathrm{CH} H \mathrm{Ph}), 6.45(\mathrm{~d}, J=7.5,1 \mathrm{H}, \mathrm{Ar}-\mathrm{H})$, 6.94-7.40 (m, 8H, Ar-H); ${ }^{13} \mathrm{C}$ NMR (trans, $100 \mathrm{MHz}, \mathrm{CDCl}_{3}$ ): 13.3, 21.0, 28.8, 37.9, 109.0, 109.6, 122.2, 123.5, 126.5, 128.6, 128.7, 131.7, 139.7, 142.1, 154.2; MS (EI) m/z (\%): 265 $\left(\mathrm{M}^{+}, 65\right), 224$ (25), 175 (55), 130 (100), 115 (50), 103 (90); HRMS :M+H, found: 266.11833. $\mathrm{C}_{16} \mathrm{H}_{16} \mathrm{NO}_{2}$ requires 266.11810 .

\section{endo-3-(1,1a,6,6a-Tetrahydro-cyclopropainden-1-yl)-3H-benzoxazolin-2-one 65}

Pale pink needles; Mp 185-186 ${ }^{\circ} \mathrm{C}\left(\mathrm{CH}_{2} \mathrm{Cl}_{2}\right.$-Petrol); IR (film): $v_{\max } 3042$ (w), 3018 (w), 2926 (w), 2853 (w), 1771 (s), 1611 (w) cm ${ }^{-1}$; ${ }^{\mathrm{H}} \mathrm{NMR}$ (endo, $\left.400 \mathrm{MHz}, \mathrm{CDCl}_{3}\right): 2.35-2.42(\mathrm{~m}, 1 \mathrm{H}$, $\left.\mathrm{CH}_{2} \mathrm{CH}\right), 3.06(\mathrm{t}, J=6.8,1 \mathrm{H}, \mathrm{ArCH}), 3.12-3.22\left(\mathrm{~m}, 3 \mathrm{H}, \mathrm{CH}_{2}\right.$ and $\left.\mathrm{CHN}\right), 6.82-6.91(\mathrm{~m}, 5 \mathrm{H}, \mathrm{Ar}-$ $H), 6.94-6.99(\mathrm{~m}, 1 \mathrm{H}$, Ar- $H), 7.05(\mathrm{~d}, J=7.5,1 \mathrm{H}, \mathrm{Ar}-H), 7.34(\mathrm{~d}, J=6.8,1 \mathrm{H}, \mathrm{Ar}-H) ;{ }^{13} \mathrm{C}$ NMR 
(endo, $100 \mathrm{MHz}, \mathrm{CDCl}_{3}$ ): 21.8, 29.5, 32.0, 33.0, 109.6, 109.8, 122.2, 123.1, 123.4, 125.7 , 126.1, 126.6, 131.0, 137.7, 142.3, 143.1, 154.5. MS (EI) $\mathrm{m} / \mathrm{z}(\%): 263(\mathrm{M}+, 65), 192(30), 148$ (40), 129 (100), 115 (50), 102 (32); HRMS: $\mathrm{M}^{+}$, found: 263.09589. $\mathrm{C}_{17} \mathrm{H}_{13} \mathrm{NO}_{2}$ requires 263.09462; Anal Calcd for $\mathrm{C}_{17} \mathrm{H}_{13} \mathrm{NO}_{2} ; \mathrm{C}, 77.55 ; \mathrm{H}, 4.98 ; \mathrm{N}, 5.32$. Found: C, 77.10; H, 4.89; N, 5.28 .

\section{trans-1-(2-(Hydroxymethyl)cyclopropyl)pyrrolidin-2-one 67}

Colourless oil; IR (film): $v_{\max } 3297$ (br), 2880, 1674 (s) $\mathrm{cm}^{-1} ;{ }^{1} \mathrm{H}$ NMR (trans, $300 \mathrm{MHz}$, $\mathrm{CDCl}_{3}$ ): 0.72 (ddd, $J=7.5,6.2,5.9,1 \mathrm{H}, \mathrm{CH} \mathrm{HCHN}$ ), 0.90 (ddd, $J=9.5,5.9,4.3,1 \mathrm{H}$, $\mathrm{CHHCHN}$ ), 1.28-1.40 (tddd, J=9.5, 6.2, 5.2, 3.5, 1H, $\left.\mathrm{CHCH}_{2} \mathrm{OH}\right), 1.90-2.03(\mathrm{~m}, 2 \mathrm{H}$, $\mathrm{CH}_{2} \mathrm{CH}_{2} \mathrm{~N}$ ), 2.32-2.41 (m, 3H, $\mathrm{CHN}$ and $\mathrm{CH}_{2} \mathrm{CO}$ ), 3.15 (dd, $\left.J=10.9,9.5,1 \mathrm{H}, \mathrm{CHHOH}\right), 3.26-$ $3.38\left(\mathrm{~m}, 2 \mathrm{H}, \mathrm{CH}_{2} \mathrm{~N}\right), 3.85(\mathrm{dd}, J=10.9,5.2,1 \mathrm{H}, \mathrm{CH} \mathrm{HOH}) ;{ }^{3} \mathrm{C} \mathrm{NMR}$ (trans, $\left.75 \mathrm{MHz}, \mathrm{CDCl}_{3}\right)$ : 9.3, 17.8, 22.6, 30.2, 31.3, 47.3, 64.5, 177.0; MS (EI) $\mathrm{m} / \mathrm{z}(\%): 156(\mathrm{M}+\mathrm{H}, 10), 138(35), 124$ (100), 96 (35), 86 (25); HRMS: $\mathrm{M}^{+}$, found: 155.09480. $\mathrm{C}_{8} \mathrm{H}_{13} \mathrm{NO}_{2}$ requires 155.09462.

\section{( \pm -1-((1R,2R,6S,7S)-2-Ethoxybicyclo[4.1.0]heptan-7-yl)pyrrolidin-2-one 69}

White solid; Mp 95-97 ${ }^{\circ} \mathrm{C}\left(\mathrm{CH}_{2} \mathrm{Cl}_{2}\right.$-Petrol); IR (film): $v_{\max } 2936$ (s), 2864 (s), 1682 (s) $\mathrm{cm}^{-1}$; ${ }^{1} \mathrm{H}$ NMR (exo, $\left.500 \mathrm{MHz}, \mathrm{CDCl} 3\right)$ : 0.97-1.06 (m, 1H, CHHCHOEt), 1.06-1.13 (m, 1H, $\mathrm{CHHCH}_{2} \mathrm{CHOEt}$ ), 1.17 (t, J=6.9, 3H, Me), 1.30-1.36 (m, 1H, $\mathrm{CHHCH}_{2} \mathrm{CHOEt}$ ), 1.38-1.48 $(\mathrm{m}, 2 \mathrm{H}, \mathrm{CHCHN}$ and $\mathrm{CHCHOEt}), 1.51-1.62(\mathrm{~m}, 2 \mathrm{H}, \mathrm{CH} H \mathrm{CHOEt}$ and CHHCHCHN), 1.80$1.98\left(\mathrm{~m}, 3 \mathrm{H}, \mathrm{CH}_{2} \mathrm{CH}_{2} \mathrm{~N}\right.$ and $\mathrm{CHHCHCHN}$ ), 2.29 (t, $J=8.2,2 \mathrm{H}, \mathrm{CH}_{2} \mathrm{CON}$ ), 2.51 (br t, $J=3.5$, $\left.1 \mathrm{H}, \mathrm{CHN}), 3.26 \mathrm{br} \mathrm{t}, J=6.8,2 \mathrm{H}, \mathrm{CH}_{2} \mathrm{~N}\right), 3.45(\mathrm{dq}, J=15.8,6.9,1 \mathrm{H}, \mathrm{CH} H \mathrm{H}), 3.73(\mathrm{~m}, 2 \mathrm{H}$, $\mathrm{CH} H \mathrm{O}$ and $\mathrm{CHO}) ;{ }^{13} \mathrm{C}$ NMR (exo, $\left.125 \mathrm{MHz}, \mathrm{CDCl}_{3}\right): 15.5,17.6,19.9,20.5,21.2,21.9,28.0$, 31.7, 35.1, 47.5, 63.0, 72.1. 175.7; MS (FAB) m/z (\%): $224\left(\mathrm{M}^{+}, 48\right), 178$ (100) ; HRMS: $\mathbf{M}^{+}$, found: $224.16480 . \mathrm{C}_{13} \mathrm{H}_{21} \mathrm{NO}_{2}$ requires 224.16505 .

\section{2-Ethoxycyclohex-1-ene $70^{23}$}

Sodium hydride (60\% dispersion in mineral oil, $954 \mathrm{mg}, 23.9 \mathrm{mmol})$ was added portionwise to a solution of 2-cyclohexen-1-ol $(2.21 \mathrm{~g}, 21.7 \mathrm{mmol})$ in anhydrous dimethylformamide (15 $\mathrm{mL}$ ) under nitrogen. After the addition was complete, the reaction was cooled to $0{ }^{\circ} \mathrm{C}$ and iodoethane $(5.34 \mathrm{~mL}, 65.0 \mathrm{mmol})$ was added dropwise (CAUTION: Exothermic). The reaction mixture was then allowed to stir at room temperature for 2 hours. Water $(50 \mathrm{~mL})$ was added and the aqueous mixture extracted with ether $(2 \times 80 \mathrm{~mL})$. The combined organic layers were washed with brine $(50 \mathrm{~mL})$, dried $\left(\mathrm{MgSO}_{4}\right)$ and concentrated. The residue was purified by column chromatography $\left(9: 1 \mathrm{Petrol}: \mathrm{Et}_{2} \mathrm{O}\right)$ to give the ether as a colourless liquid $(620 \mathrm{mg})$. Elution of the column with 1:1 Et ${ }_{2} \mathrm{O}:$ Petrol gave the starting alcohol $(660 \mathrm{mg})$; IR (film): $v_{\max } 3028,2922(\mathrm{~s}), 2655$ (s), 1508, 1456 (s) cm ${ }^{-1} ;{ }^{1} \mathrm{H}$ NMR $\left(500 \mathrm{MHz}, \mathrm{CDCl}_{3}\right): 1.21$ (t, $J=6.9,3 \mathrm{H}, \mathrm{Me}), 1.51-1.58(\mathrm{~m}, 1 \mathrm{H}), 1.67$ (dddd, $1 \mathrm{H}, J=12.0,9.1,6.3,2.5), 1.72-1.86(\mathrm{~m}$, $2 \mathrm{H}), 1.91-1.98(\mathrm{~m}, 1 \mathrm{H}), 2.00-2.08(\mathrm{~m}, 1 \mathrm{H}), 3.51(\mathrm{dq}, J=9.1,6.9,1 \mathrm{H}, \mathrm{CHHMe}), 3.57(\mathrm{dq}$, $J=9.1,6.9,1 \mathrm{H}, \mathrm{CH} H \mathrm{Me}), 3.81-3.86(\mathrm{~m}, 1 \mathrm{H}, \mathrm{CHO}), 5.75-5.79(\mathrm{~m}, 1 \mathrm{H}, H \mathrm{C}=\mathrm{CH}), 5.81-5.86$ $(\mathrm{m}, 1 \mathrm{H}, \mathrm{HC}=\mathrm{CH}) ;{ }^{3} \mathrm{C}$ NMR $\left(125 \mathrm{MHz}, \mathrm{CDCl}_{3}\right): 15.8,19.3,25.2,28.4,63.4,72.6,128.0$, 130.6 .

\section{2-(2-tert-Butylcyclopropylamino)ethanol 71}

Lithium hydroxide monohydrate $(1.24 \mathrm{~g}, 29.5 \mathrm{mmol}, 30 \mathrm{eq})$ was added in one portion to a suspension of cyclopropane $57(0.18 \mathrm{~g}, 0.98 \mathrm{mmol}, 1 \mathrm{eq})$ in a mixture of absolute ethanol (14 $\mathrm{mL})$ and water $(6 \mathrm{~mL})$. The reaction mixture was heated at reflux for $20 \mathrm{~h}$ and then allowed to cool to room temperature. Most of the ethanol was removed in vacuo and saturated aqueous ammonium chloride $(5 \mathrm{~mL})$ was added to the residue. The aqueous layer was extracted with dichloromethane $(2 \times 10 \mathrm{~mL}$, then $2 \times 5 \mathrm{~mL})$ and the combined organic extracts were dried $\left(\mathrm{Na}_{2} \mathrm{SO}_{4}\right)$, filtered and concentrated to give the amino alcohol 142 (75 mg, $\left.0.48 \mathrm{mmol}, 49 \%\right)$ as a yellow oil; IR (film): $v_{\max } 3300(\mathrm{br}), 2953(\mathrm{~s}), 2866(\mathrm{~m}), 1468(\mathrm{~m}) \mathrm{cm}^{-1} ;{ }^{1} \mathrm{H}$ NMR $(500$ $\left.\mathrm{MHz}, \mathrm{CDCl}_{3}\right): \delta 0.34-0.41\left(\mathrm{~m}, 2 \mathrm{H}, \mathrm{CH}_{2} \mathrm{CHN}\right), 0.59$ (ddd, J=9.9, 6.2, 3.5, 1H, CHCHN), 0.77 $(\mathrm{s}, 9 \mathrm{H}, 3 \times \mathrm{Me}), 1.95\left(\mathrm{dt}, J=7.2,3.5,1 \mathrm{H}_{\mathrm{p}} \mathrm{CHN}\right), 2.71-2.77\left(\mathrm{~m}, 2 \mathrm{H}, \mathrm{CH}_{2} \mathrm{~N}\right), 2.90(\mathrm{br} \mathrm{s}, 2 \mathrm{H}, \mathrm{OH}$ and $\mathrm{NH}), 3.57\left(\mathrm{t}, J=5.5,2 \mathrm{H}, \mathrm{CH}_{2} \mathrm{OH}\right) ;{ }^{13} \mathrm{C} \mathrm{NMR}\left(100 \mathrm{MHz} \mathrm{CDCl}_{3}\right): \delta 9.4,28.5,28.9,31.8$, 33.3, 51.1, 60.5; MS (CI) $\mathrm{m} / z(\%): 158(\mathrm{M}+\mathrm{H}, 100), 100(86)$; HMRS: $\mathrm{M}+\mathrm{H}$, found 158.154382. $\mathrm{C}_{9} \mathrm{H}_{20} \mathrm{NO}$ requires 158.15448 .

tert-Butyl $N$-[(2-tert-butylcyclopropyl)-(2-hydroxyethyl)]carbamate 72 
Di-tert-butyl dicarbonate $(106 \mathrm{mg}, 0.49 \mathrm{mmol}, 1.2 \mathrm{eq})$ was added portionwise to a solution of amino alcohol $142(64 \mathrm{mg}, 0.407 \mathrm{mmol}, 1 \mathrm{eq})$ and triethylamine $(0.07 \mathrm{~mL}, 0.51 \mathrm{mmol}, 1.25$ eq) in dry dichloromethane $(2 \mathrm{~mL})$ under nitrogen. After stirring for $24 \mathrm{~h}$, the reaction mixture was concentrated in vacuo. The crude product was purified by flash column chromatography (Petrol:EtOAc 4:1 to 3:1) to give the title compound 143 (76 mg, $0.295 \mathrm{mmol}, 72 \%$ ) as a colourless oil; IR (film): $v_{\max } 3450$ (br), $2955(\mathrm{~s}), 2870(\mathrm{~m}), 1685$ (s) $\mathrm{cm}^{-1} ;{ }^{1} \mathrm{H}$ NMR (500 $\left.\mathrm{MHz}, \mathrm{CDCl}_{3}\right): \delta 0.65$ (ddd, $\left.J=9.9,5.8,3.9,1 \mathrm{H}, \mathrm{CHHCHN}\right), 0.71$ (ddd, $J=7.5,6.8,5.8,1 \mathrm{H}$, $\mathrm{CH} H \mathrm{CHN}), 0.82(\mathrm{~s}, 9 \mathrm{H}, 3 \times \mathrm{Me}), 0.85$ (ddd, $J=9.9,6.8,3.9,1 \mathrm{H}, \mathrm{CHCHN}), 1.42(\mathrm{~s}, 9 \mathrm{H}$, $\left.\mathrm{OC}\left(\mathrm{CH}_{3}\right)_{3}\right), 2.45(\mathrm{dt}, J=7.5,3.9,1 \mathrm{H}, \mathrm{CHN}), 3.29$ (ddd, $\left.J=14.6,6.2,4.6,1 \mathrm{H}, \mathrm{CHHN}\right), 3.41$ (ddd, $J=14.6,6.5,4.5,1 \mathrm{H}, \mathrm{CH} H \mathrm{~N}), 3.57\left(\mathrm{~m}, 2 \mathrm{H}, \mathrm{CH}_{2} \mathrm{OH}\right) ;{ }^{13} \mathrm{C} \mathrm{NMR}\left(125 \mathrm{MHz}, \mathrm{CDCl}_{3}\right): \delta$ 12.2, 28.4, 28.4, 29.1, 32.1, 32.2, 50.7, 62.4, 80.1, 158.2; MS (CI+) $m / z(\%): 256$ (M-H, 100), 200 (30), 182 (32), 156 (45), 147 (65), 138 (29); HMRS: M-H, found 256.191464. $\mathrm{C}_{14} \mathrm{H}_{26} \mathrm{NO}_{4}$ requires 256.19126 .

\section{[tert-Butoxycarbonyl-(2-tert-butylcyclopropyl)-amino]acetic acid 73}

An aqueous solution of sodium hydroxide $(0.5 \mathrm{M}$ in water, $2.2 \mathrm{~mL}, 1.1 \mathrm{mmol}, 4 \mathrm{eq})$ and potassium permanganate $(0.65 \mathrm{M}$ in water, $1.7 \mathrm{~mL}, 1.1 \mathrm{mmol}, 4 \mathrm{eq})$ were added successively to a solution of alcohol $143(70 \mathrm{mg}, 0.27 \mathrm{mmol}, 1 \mathrm{eq})$ in tert-butanol $(2.7 \mathrm{~mL})$. The reaction mixture was stirred for $24 \mathrm{~h}$ and then quenched with sodium thiosulfate $(5 \%$ in water, 7.75 $\mathrm{mL})$. Diethyl ether $(10 \mathrm{~mL})$ was added and the mixture was transferred into a separating funnel. The aqueous layer was separated and then acidified to $\mathrm{pH} 2$ with an aqueous hydrochloric acid solution $(1 \mathrm{M})$ at $4{ }^{\circ} \mathrm{C}$. The mixture was then extracted with EtOAc $(3 \times 10$ $\mathrm{mL}$ ) and the combined organic extracts were dried $\left(\mathrm{Na}_{2} \mathrm{SO}_{4}\right)$, filtered and concentrated in vacuo to give the title compound $144(62 \mathrm{mg}, 0.23 \mathrm{mmol}, 84 \%)$ as a white amorphous solid; IR (film): $v_{\max } 3450$ (br), 2954 (m), 1699 (s) cm ${ }^{-1} ;{ }^{1} \mathrm{H} \mathrm{NMR}\left(500 \mathrm{MHz}, 328 \mathrm{~K}, \mathrm{CDCl}_{3}\right): \delta 0.71$ (ddd, $J=9.9,5.7,3.9,1 \mathrm{H}, \mathrm{CHHCHN}), 0.75$ (ddd, $J=7.4,6.7,5.7,1 \mathrm{H}, \mathrm{CH} H \mathrm{CHN}), 0.84$ (s, 9H, $3 \times \mathrm{Me}), 0.87(\mathrm{ddd}, J=9.9,6.7,3.9,1 \mathrm{H}, \mathrm{CHCHN}), 1.44\left(\mathrm{~s}, 9 \mathrm{H}, \mathrm{OC}\left(\mathrm{CH}_{3}\right)_{3}\right), 2.59(\mathrm{dt}, J=7.4$, $3.9,1 \mathrm{H}, \mathrm{CHN}), 3.83(\mathrm{~d}, J=17.7,1 \mathrm{H}, \mathrm{CHHN}), 4.04(\mathrm{~d}, J=17.7,1 \mathrm{H}, \mathrm{CH} H \mathrm{~N}), 8.73(\mathrm{br} \mathrm{s}, 1 \mathrm{H}$, $\mathrm{OH}) ;{ }^{13} \mathrm{C}$ NMR $\left(125 \mathrm{MHz}, 328 \mathrm{~K}, \mathrm{CDCl}_{3}\right): \delta 12.2,28.4,28.4,29.0,32.3,32.5,50.0,80.7$, 156.7, 175.3; MS (CI+) m/z (\%): 272 (M+H, 33), 216 (92), 172 (52), 154 (100); HMRS: $\mathrm{M}+\mathrm{H}$, found $272.18676 . \mathrm{C}_{14} \mathrm{H}_{26} \mathrm{NO}_{4}$ requires 272.18617 .

\section{2-(1,1a,6,6a-Tetrahydro-cyclopropa[a]inden-1-ylamino)-phenol 74}

Lithium triethylborohydride (1.0M in THF, $0.75 \mathrm{~mL}, 0.75 \mathrm{mmol}$ ) was added dropwise to a solution of cyclopropane 65 (39 mg, $0.15 \mathrm{mmol})$ in THF $(1 \mathrm{~mL})$ under nitrogen. The reaction mixture was stirred at room temperature overnight and then quenced with saturated ammonium chloride $(4 \mathrm{~mL})$. The aqueous mixture was extracted with ethyl acetate $(3 \times 10$ $\mathrm{mL})$, and the combined organic layers washed with brine $(10 \mathrm{~mL})$, dried $\left(\mathrm{MgSO}_{4}\right)$ and concentrated. The residue was purified by column chromatography (1:9 EtOAc:Petrol then 3:7 then 1:1) to give the catecolamine $\mathbf{7 4}$ as a pale pink solid (12 mg, 34\%); IR (film): $v_{\max }$ 3150-3400 (br), 2924, 1659 (s), $1597 \mathrm{~cm}^{-1}$; ${ }^{1} \mathrm{H}$ NMR (trans, $500 \mathrm{MHz}, \mathrm{CDCl}_{3}$ ): 2.27 (br q, $J=6.7,1 \mathrm{H}, \mathrm{CHCH} \mathrm{CHr}_{2}, 2.50(\mathrm{~d}, J=18.0,1 \mathrm{H}, \mathrm{ArCHH}), 3.05(\mathrm{dd}, J=8.4,7.0,1 \mathrm{H}, \mathrm{CHAr}), 3.12$ (dd, $J=18.0,7.0,1 \mathrm{H}, \operatorname{ArCH} H), 3.73(\mathrm{t}, J=7.0,1 \mathrm{H}, \mathrm{CHNH}), 6.23($ br s, $1 \mathrm{H}, \mathrm{NH}), 6.76-7.30$ (m, $8 \mathrm{H}, \mathrm{Ar}-\mathrm{H}), 8.14$ (br s, $1 \mathrm{H}, \mathrm{OH}) ;{ }^{13} \mathrm{C}$ NMR (trans, $125 \mathrm{MHz}, \mathrm{CDCl}_{3}$ ): 23.4, 31.3, 31.8, 39.3, $119.6,120.5,122.5,124.5,124.6,126.5,127.0,127.8,137.7,142.7,150.6,165.9$; MS (CI+) $m / z(\%): 266(100), 238(23, \mathrm{M}+\mathrm{H}), 166(23)$; HRMS: $\mathrm{M}+\mathrm{H}$, found: $238.12343 . \mathrm{C}_{16} \mathrm{H}_{16} \mathrm{NO}$ requires 238.12318 .

\subsubsection{1-[2-(Hydroxycyclopropyl)]-2-pyrrolidinone 75}

Tetrabutylammonium fluoride (1 M solution in THF, $1.09 \mathrm{~mL}, 1.09 \mathrm{mmol}$ ) was added to a solution of cyclopropylsilane $\mathbf{5 0}(0.54 \mathrm{mmol})$ in THF $(0.3 \mathrm{~mL})$ under nitrogen. After $30 \mathrm{~min}$ of stirring, methanol $(1.4 \mathrm{~mL})$, potassium hydrogencarbonate $(0.11 \mathrm{~g}, 1.09 \mathrm{mmol})$ and hydrogen peroxide ( $30 \%$ solution in water, $1.12 \mathrm{~mL}, 10.9 \mathrm{mmol}$ ) were added to the solution. The reaction mixture was stirred for $18 \mathrm{~h}$ and then sodium thiosulfate pentahydrate $(2.97 \mathrm{~g}$, $11.97 \mathrm{mmol}$ ) was added. After stirring for $30 \mathrm{~min}$ the mixture was filtered and concentrated in vacuo. The crude product was then purified by flash column chromatography to give the desired amino cyclopropanol as a colourless oil (35\%); IR (film): $v_{\max } 3369$ (br), 2989 (w), $2957(\mathrm{w}), 2859$ (w), 1662 (s), 1463, $1425 \mathrm{~cm}^{-1}$; ${ }^{1} \mathrm{H}$ NMR (500 MHz, $\mathrm{CDCl}_{3}$ ): $\delta 0.83$ (ddd, $J=7.5,7.1,5.0,1 \mathrm{H}, \mathrm{CHHCHN}), 1.02$ (ddd, $J=8.7,7.1,4.1,1 \mathrm{H}, \mathrm{CH} H \mathrm{CHN}), 1.85-1.99$ (m, 2H, $\left.\mathrm{CH}_{2} \mathrm{CH}_{2} \mathrm{~N}\right), 2.33\left(\mathrm{t}, J=8.1,2 \mathrm{H}, \mathrm{CH}_{2} \mathrm{CO}\right), 2.53(\mathrm{ddd}, J=8.7,5.0,1.6,1 \mathrm{H}, \mathrm{CHN}), 3.20-3.30(\mathrm{~m}$, $\left.2 \mathrm{H}, \mathrm{CH}_{2} \mathrm{~N}\right), 3.50(\mathrm{ddd}, J=7.5,4.1,1.6,1 \mathrm{H}, \mathrm{CHOH}), 4.31($ br s, $1 \mathrm{H}, \mathrm{OH}) ;{ }^{13} \mathrm{C} \mathrm{NMR}(125$ 
$\left.\mathrm{MHz}, \mathrm{CDCl}_{3}\right): \delta 14.6,17.9,31.6,32.4,47.4,51.1,176.6 ; \mathrm{MS}(\mathrm{EI}) \mathrm{m} / z(\%): 141\left(\mathrm{M}^{+}, 7\right), 112$ (100), 98 (24), 84 (23), 69 (47); HMRS: $\mathrm{M}^{+}$, found 141.07832. $\mathrm{C}_{7} \mathrm{H}_{11} \mathrm{NO}_{2}$ requires 141.07843.

\section{References}

1. Salaün, J. Top. Curr. Chem. 2000, 207, 1-67; Salaün, J.; Baird, M. S. Curr. Med. Chem. 1995, 2, 511-542; Gnad, F.; Reiser, O. Chem. Rev. 2003, 103, 1603-1623; Stammer, C. H. Tetrahedron, 1990, 46, 2231-2254; Burgess, K.; Ho, K. -K.; Moye-Sherman, D. Synlett, 1994, 575-583.

2. Vilsmaier, E. The Chemistry of the Cyclopropyl Group; Rappoport Z., Ed, John Wiley \& Sons: Chichester, 1987, p 1341.

3. Asunskis, J.; Shechter, H. J. Org. Chem. 1968, 33, 1164-1168; Wurz, R. P.; Charette, A. B. J. Org. Chem. 2004, 69, 1262-1269.

4. Kang, J.; Kim, K. S. J. Chem. Soc. Commun. 1987, 897-898.

5. Aggarwal, V. K.; de Vicente, J.; Bonnert, R. V. Org. Let. 2001, 3, 2785-2788; Tsai, C. -C.; Hsieh, I. -L.; Cheng, T. -T.; Tsai, P. -K.; Lin, K -W.; Yan, T. -H. Org. Let. 2006, 8, 2261-2263.

6. de Meijere, A.; Kozhushkov, S. I.; Savchenko, A. I. J. Organomet. Chem. 2004, 689, 20332055.

7. Brookhart, M.; Studabaker, W. B. Chem. Rev. 1987, 87, 411-432; Merino, I.; Hegedus, L. S. Organometallics 1995, 14, 2522-2531; Barluenga, J.; López, S.; Trabanco, A. A. ; Flórez, J. Chem. Eur. J. 2001, 7, 4723-4729; Barluenga, J.; Aznar, F.; Gutiérrez, I.; García-Granda, S.; Llorca-Baragaño, M. A. Org. Let. 2002, 4, 4273-4276.

8. Motherwell, W. B.; Roberts, L. R. J. Chem. Soc. Commun. 1992, 1582-1583; Motherwell, W. B.; Roberts, L. R. Tet. Let. 1995, 36, 1121-1124; Motherwell, W. B. J. Organomet. Chem. 2001, 624, 41-46; Motherwell, W. B. Pur. Appl. Chem. 2002, 74, 135-142.

9. Motherwell, W. B.; O’ Mahony, D. J. R.; Popkin, M. E. Tet. Let. 1998, 39, 5285-5288.

10. Fletcher, R. J.; Motherwell, W. B.; Popkin, M. E. Chem. Comm. 1998, 2191-2192.

11. Bégis, G.; Cladingboel, D. E.; Motherwell, W. B. Chem. Comm. 2003, 2656-2657.

12. Bégis, G.; Cladingboel, D. E.; Motherwell, W. B.; Sheppard, T. D.; Tocher, D. A. Synthesis 2005, 3186-3188.

13. Gmeimer, P.; Bollinger, B. Synthesis 1995, 168-170

14. Norman, M. H.; Minick, D. J.; Rigdon, G. C. J. Med. Chem. 1996, 39, 149-157.

15. Chan, J. H. -H.; Rickborn, B. J. Am. Chem. Soc. 1968, 90, 6406-6411; Denmark, S. E.; Edwards, J. P. J. Org. Chem. 1991, 56, 6974-6981.

16. Tanaka, H.; Ogasawara, K. Tet. Let. 2002, 43, 4417-4420.

17. Koldobskii, A. B.; Vakhmistrov, V. E.; Solodova, E. V.; Shilova, O. S.; Kalinin, V. N. Dokl. Chem. Eng. Trans. 2002, 387, 289-292.

18. Treppendahl, S.; Jakobsen, P. Acta. Chem. Scand. Ser. B. 1982, 36, 275-276.

19. Molander, G. A.; Harris, C. R. J. Org. Chem. 1997, 62, 2944-2956.

20. Troxler, T.; Scheffold, R. Helv. Chim. Act. 1994, 77, 1193-1202.

21. Inoue, S. -I.; Takaya, H.; Tani, K.; Otsuka, S.; Sato, T.; Noyori, R. J. Am. Chem. Soc. 1990, 112, 4897-4905. 
22. The alkene 36 was purchased from the Aldrich chemical company (cat number 35,773-1) but is no longer commercially available.

23. Baguley, P. A.; Walton, J. C. J. Chem. Soc. Perkin Trans. I 1998, 2073-2082. 